\title{
Microfluidic bio-particle manipulation for biotechnology
}

\author{
Barbaros Çetin ${ }^{\mathrm{a}, *}$, Mehmet Bülent Özer ${ }^{\mathrm{b}}$, Mehmet Ertuğrul Solmaz ${ }^{\mathrm{c}, \mathrm{d}}$ \\ a Mechanical Engineering Department, Microfluidics E Lab-on-a-Chip Research Group, Ihsan Dog̃ramacı Bilkent University, Ankara 06800, Turkey \\ ${ }^{\mathrm{b}}$ Department of Mechanical Engineering, TOBB University of Economics and Technology, Ankara 06560, Turkey \\ ${ }^{\mathrm{c}}$ Department of Electrical and Electronics Engineering, Izmir Katip Çelebi University, İzmir 35620, Turkey \\ d National Nanotechnology Research Center, Ankara 06800, Turkey
}

\section{A R T I C L E I N F O}

\section{Article history:}

Received 23 March 2014

Received in revised form 8 July 2014

Accepted 14 July 2014

Available online 21 July 2014

\section{Keywords:}

Biomedical

Bioprocess design

Bioseparations

Fluid mechanics

Microfluidics

Bio-particle manipulation

\begin{abstract}
A B S T R A C T
Microfluidics and lab-on-a-chip technology offers unique advantages for the next generation devices for diagnostic therapeutic applications. For chemical, biological and biomedical analysis in microfluidic systems, there are some fundamental operations such as separation, focusing, filtering, concentration, trapping, detection, sorting, counting, washing, lysis of bio-particles, and PCR-like reactions. The combination of these operations led to the complete analysis systems for specific applications. Manipulation of the bio-particles is the key ingredient for these applications. Therefore, microfluidic bio-particle manipulation has attracted a significant attention from the academic community. Considering the size of the bio-particles and the throughput of the practical applications, manipulation of the bio-particles is a challenging problem. Different techniques are available for the manipulation of bio-particles in microfluidic systems. In this review, some of the techniques for the manipulation of bio-particles; namely hydrodynamic based, electrokinetic-based, acoustic-based, magnetic-based and optical-based methods have been discussed. The comparison of different techniques and the recent applications regarding the microfluidic bio-particle manipulation for different biotechnology applications are presented. Finally, challenges and the future research directions for microfluidic bio-particle manipulation are addressed.
\end{abstract}

(c) 2014 Elsevier B.V. All rights reserved.

\section{Introduction}

The miniaturization trend of integrated circuits since 1970s, and the development of advanced fabrication techniques for micro and nano-scale devices [1] since 1980s led to the usage of devices having the dimensions of micrometers and nanometers in many fields. This trend has helped microfluidics, which is the flow physics at micro scale, become an active research area at the intersection of chemistry, physics, biology and engineering. This intersection eliminated the boundaries between these disciplines. The elimination of these boundaries has posed many challenges and new directions for organizations of education and research. One of the important challenges is the rapid development of biochips, miniaturized analysis systems or lab-on-a-chip (LOC) devices which are microfluidic platforms on which one can handle chemical and biological analyses, point-of-care testing, clinical and forensic analysis, molecular and medical diagnostics for biological, biomedical and

\footnotetext{
* Corresponding author. Tel.: +90 312290 2108; fax: +90 3122664126

E-mail addresses: barbaros.cetin@bilkent.edu.tr, barbaroscetin@gmail.com (B. Çetin), bulent.ozer@gmail.com (M.B. Özer), mehmete.solmaz@ikc.edu.tr (M.E. Solmaz).
}

chemical applications. LOC devices can perform the same specialized functions as their bench-top counterparts. They can also perform clinical diagnoses, scan DNA, run electrophoretic separations, act as microreactors, detect cancer cells and identify bacteria and viruses [2]. On a single chip, hundreds of different reactions and/or analyses can be performed at the same time through hundreds of parallel microchannels. Originally it was thought that the most significant benefit of these LOC devices would have been the analytical improvements associated with the scaling down of the size. Further developments revealed other significant advantages such as: (i) small amount of sample (in the nano to picoliter range, opening the door to the possibility of analyzing components from single cells), (ii) small amount of reagents, (iii) very short reaction and analysis time compared to bench-top counterparts, (iv) reduced manufacturing costs, (v) increased automation, (vi) high portability, and (vii) opportunity for massively parallel chemical analyses either on the same or multiple samples [3].

For chemical, biological and biomedical analyses in microfluidic systems, there are some fundamental operations such as separation, focusing, filtering, concentration, trapping, sorting, detection, counting, washing, lysis of bio-particles, and PCR-like reactions. The combination of these operations led to the complete analysis system or LOC system for a certain application. 
Manipulation of the bio-particles is the key ingredient for the aforementioned operations. Therefore, microfluidic bio-particle manipulation has attracted significant attention from the academic community. Considering the size of the bio-particles and the required throughput for the practical applications, manipulation of the bio-particles is a challenging problem. Many research groups and scientists have proposed different techniques to manipulate bio-particles such as hydrodynamic-based, electrokinetic-based, acoustic-based, magnetic-based, optical-based etc. In this review, these different techniques are discussed. Moreover, the comparison of different techniques and the recent biotechnology applications regarding the microfluidic bio-particle manipulation are presented. Finally, challenges and the future research directions are also addressed.

\section{Manipulation methods}

Manipulation methods can be categorized as passive or active methods depending on the presence of an external force field. Passive systems utilize the flow field together with the channel geometry or topology changes to manipulate the motion of particles. On the other hand, active systems utilize an external force field such as electric, acoustic, magnetic and optic to manipulate the motion of particles. These methods can also be categorized as labelbased or label-free methods depending on the need for any labeling (or tags) for the bio-particles. The label-free methods utilize the intrinsic properties of the bio-particles such as size, shape, density, dielectric properties, acoustic properties and refractive index. On the other hand, the label-based techniques require additional labels to manipulate bio-particles. As an example, two conventional cell sorting technique namely fluorescence-activated cell sorting (FACS) and magnetic-activated cell sorting (MACS) require cellspecific labeling through fluorophore-conjugated antibodies and magnetic beads conjugated with antibodies, respectively [4].

Considering the manipulation of a bio-particle in a microfluidic system, depending on the methods there may exist multiple forces on a bio-particle, some of which can be dominant or negligible. Therefore, the order of magnitude estimate of the various forces experienced by a bio-particle is crucial for microfluidic applications to predict the resultant motion of bio-particles. As an example, Brownian motion is the random movement of particles due to the thermal effects; however, Brownian motion is negligible for the particles with a size larger than $1 \mu \mathrm{m}$ for microfluidic applications [5].

There are several techniques to manipulate bio-particles in microfluidic systems. Several of those methods are reviewed within this paper. Stand-alone review papers are present for each of these methods [6-14] since there has been a vast amount of research effort on these techniques for microfluidic platforms for the last two decades. In this review, our objective is to give the basics of each method. More work is dedicated for the comparison of the techniques in terms of associated sample preparation, throughput, channel geometry, material and fabrication, and the required hardware. We believe that such a comparison will provide valuable help for the researchers from many disciplines who would like to apply microfluidic technology to bio-particle related biotechnology applications.

\subsection{Hydrodynamic-based (HD)}

In microfluidic applications, the flow can be induced by pressure difference (pressure-driven flow) and/or by electrical field (electroosmotic flow). Since electric field is introduced for electro-osmotic flow, other forces (which will be discussed in the following subsection) other than drag force generated on the particle come into picture. In the case of pressure-driven flow, pressure difference is the main parameter which control the incompressible fluid flow in microchannels. The drag force is the only force generated on the particles as a result of the interaction of the particle with the flow field. Hydrodynamic-based methods are passive methods in which the bio-particle manipulation is performed by use of the drag force generated on the particles through specially designed channel geometries and topologies. The dimensionless numbers which characterize the particle flow in a microchannel are the channel Reynolds number $(R e)$ and the particle Reynolds number $\left(R e_{p}\right)[15]$ :

$R e=\frac{\rho U_{\max } D_{h}}{\mu}, \quad R e_{p}=\frac{\rho U_{\max } d^{2}}{\mu D_{h}}=\operatorname{Re}\left(\frac{d}{D_{h}}\right)^{2}$,

where $U_{\max }$ is the maximum velocity in the microchannel, $\rho$ is the fluid density, $\mu$ is the dynamic fluid viscosity, $d$ is the particle diameter, and $D_{h}$ is the hydraulic diameter of the channel. Typically, flows within microchannels are in Stoke's flow regime (low Re flows) which means the flow follows the boundaries of the domain. When particles are present within the channel, they also follow the streamlines of the flow field in a deterministic manner. However, when an obstacle and/or flow contraction/expansion is presented within the channel, the particle trajectories reveal size dependence. Therefore, by specially designed channel geometries, bio-particles can be manipulated according to their size and deformability.

Introducing obstacles and posts with a critical spacing can be utilized as filter structure to capture (trap) or isolate specific bio-particle of interest with a size larger than the critical size [6]. However, pore-based filtration may be ineffective with deformable bio-particles and/or bio-particles with unique shapes. By introducing series of posts, a size dependent lateral displacement of bio-particles can also be achieved, which is known as deterministic lateral displacement (DLD) (see Fig 1a) [16-20]. DLD can be utilized for bio-particle separation, sorting and focusing. The presence of slanted or anisotropic obstacles within the microchannel can also induce size-based motion of the particles due to the particle-obstacle interaction induced rotational flows, which is known as hydrophoresis (see Fig. 1b) and can be implemented for bio-particle separation, sorting and focusing [21-26]. With the introduction of contraction/expansion (pinch segment) within the microchannel network together with the laminar flow profile, bioparticles can also be manipulated to flow at different streamlines, which is known as pinch-flow fractionation (PFF) (see Fig 1c) and can be implemented for bio-particle separation, sorting and focusing [27-31].

The Stoke's flow regime is valid up to $R e \sim 1$. When $R e$ reaches unity and beyond, the inertial effects become significant and modify the flow characteristics, which is known as inertial microfluidics. In this regime, particles do not follow the streamlines of the flow field. When the inertial effects come into picture, two inertial lift forces are induced on the particle: (i) a shear gradient lift force and (ii) a wall-effect lift force [15]. A wall-effect lift force induces a repelling force away form the wall. On the other hand, sheargradient lift force induces an attractive force towards the wall [15]. When the channel geometry becomes curved, a secondary rotational flow begins to be observed due to the inertia of the fluid which is known as Dean flow. The dimensionless numbers which characterizes this secondary flow are the Dean number $(D e)$ and the curvature ratio $(\delta)[15,32]$ :

$D e=\operatorname{Re}\left(\frac{D_{h}}{2 r}\right)^{1 / 2}, \quad \delta=\frac{D_{h}}{2 r}$,

where $r$ is the radius of curvature of the channel. De and $\delta$ are two important parameters which affect the motion of the particles within curved channels. Inertial microfluidics can be utilized for separation, sorting, focusing, and isolation of bio-particles [33-39]. 


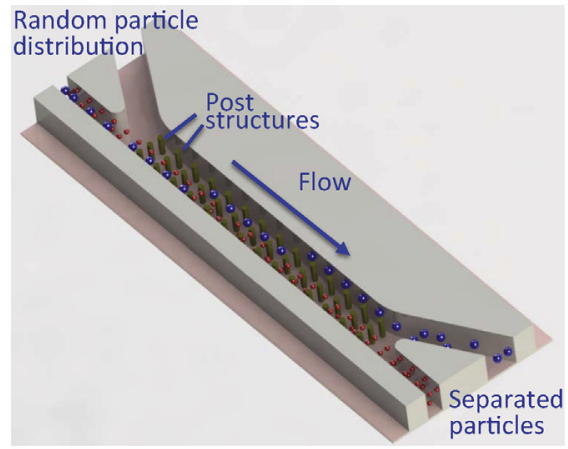

(a) DLD

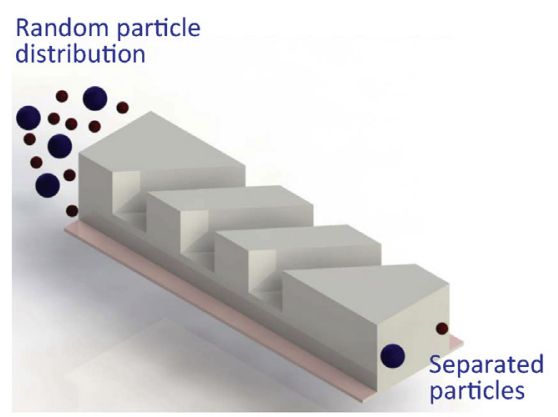

(b) Hydrophoresis

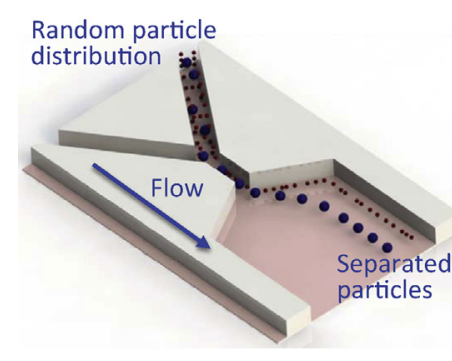

(c) PFF
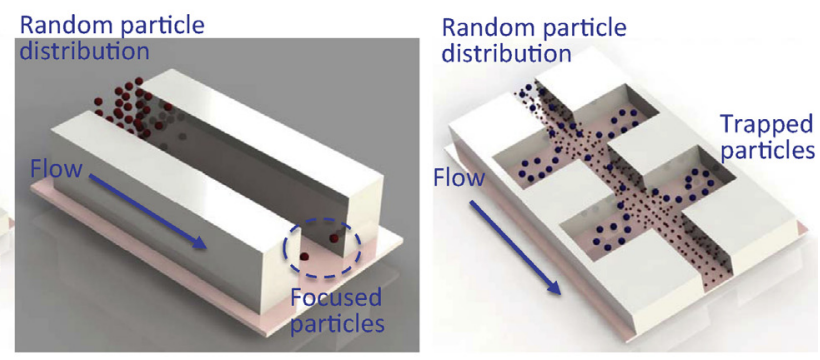

(d) Inertial microfluidics

Fig. 1. Basic principles of hydrodynamic-based methods: (a) DLD, (b) hyrdophoresis, (c) pinched flow fractionation, (d) inertial microfluidics.

A schematics of an inertial microfluidics based sorting can be seen in (see Fig 1d).

The HD manipulation can be also utilized for the separation by shape, since the trajectory of the particles within a microfluidic channel may also possess shape dependence depending on the channel geometry and characteristics of flow. More specifically, the flow of a spherical particle and non-spherical particle may differ. Separation and sorting of spherical and non-spherical particles is important for clinical applications such as separation of yeast cells at different cell stage and separation of parasites form blood. More recently, some research efforts have been focused on the implementation of HD applications on separation by shape using DLD $[19,18,20]$, PFF [28] and inertial microfluidics [40].

\subsection{Electrokinetic-based (EK)}

Electrical forces like electrophoresis (EP) and dielectrophoresis (DEP) are the subtle solutions to manipulate particles in LOC devices due to their favorable scaling for the reduced size of the system [41]. EP is the movement of the electrically-charged particles in an electrical field due to the Coulombic body force (electrophoretic force) acting on the particles because of their surface charge. For the utilization of the EP, the particle needs to be charged and the applied electric field needs to be constant or direct current (DC).

The EP force on a particle subjected to an electric field of $\mathbf{E}$ can be written as:

$\mathbf{F}_{\mathrm{EP}}=q \mathbf{E}$

where $q$ is the net charge of the particle [2]. EP is commonly used in conventional and well-developed separation techniques such as capillary electrophoresis to separate DNA and proteins.

DEP is the movement of particles in a non-uniform electric field due to the interaction of the particle's dipole and spatial gradient of the electric field. DEP is applicable even for non-conducting particles and can be generated either by using DC or alternating current (AC) field.
The DEP force on a spherical particle subjected to a DC field of $\mathbf{E}$ can be written as [8]:

$\mathbf{F}_{\mathrm{DEP}}=2 \pi \varepsilon_{m} f_{\mathrm{CM}} R^{3} \nabla(\mathbf{E} \cdot \mathbf{E})=2 \pi \varepsilon_{m} f_{\mathrm{CM}} R^{3} \nabla|\mathbf{E}|^{2}$,

where $\mathbf{E}$ is the electric field vector, $\varepsilon_{m}$ is the absolute permittivity of the suspending medium, and $R$ is the particle radius. $f_{\mathrm{CM}}$ is the Clausius-Mossotti (CM) factor, which is given by

$f_{\mathrm{CM}}=\frac{\varepsilon_{p}-\varepsilon_{m}}{\varepsilon_{p}+2 \varepsilon_{m}}$,

where $\varepsilon$ is the permittivity, and subscripts $p$ and $m$ stand for the particle and the medium, respectively. CM factor has numerical limits from -0.5 to 1.0 . For negative $C M$, negative-DEP (nDEP) force (which is in the direction of minima of the gradient of the electric field strength) is generated on the particle. For positive CM, positive-DEP ( $\mathrm{pDEP}$ ) force (which is in the direction of maxima of the gradient of the electric field strength) is generated on the particle.

Similarly, for a spherical particle in an AC-field, the timeaveraged DEP force can be expressed as [8]

$$
\left\langle\mathbf{F}_{\mathrm{DEP}}(t)\right\rangle=2 \pi \varepsilon_{m} \mathcal{R} e\left[f_{\mathrm{CM}}\right] R^{3} \nabla \mathbf{E}_{r m s}^{2},
$$

where $E_{r m s}$ is the root-mean-square of the AC-field, $\mathcal{R} e\left[f_{\mathrm{CM}}\right]$ is the real part of the Clausius-Mossotti factor which is defined as

$f_{\mathrm{CM}}\left(\tilde{\varepsilon}_{p}, \tilde{\varepsilon}_{m}\right)=\frac{\tilde{\varepsilon}_{p}-\tilde{\varepsilon}_{m}}{\tilde{\varepsilon}_{p}+2 \tilde{\varepsilon}_{m}}$,

where $\tilde{\varepsilon}$ is the complex permittivity and defined as

$\tilde{\varepsilon}=\varepsilon-j\left(\frac{\sigma}{\omega}\right)$.

Time-averaged DEP force, Eq. (6), is valid for a stationary ACfield. If the phase of the AC-field has a spatial variation, Eq. (6) needs to be modified to include this effect. In general sense, time-averaged DEP force can be written as [8]:

$\left\langle\mathbf{F}_{\mathrm{DEP}}(t)\right\rangle=2 \pi \varepsilon_{m} \mathcal{R} e\left[f_{\mathrm{CM}}\right] R^{3} \nabla \mathbf{E}_{r m s}^{2}+4 \pi \varepsilon_{m} \operatorname{Im}\left[f_{\mathrm{CM}}\right] R^{3}\left(\mathbf{E}_{r m s, i}^{2} \nabla \varphi_{i}\right)$, 
where $\varphi$ is the phase of the AC-field. Subscript $i$ refers to each component of the electric field and the phase gradient. The last term in the parenthesis is a tensor notation and refers to the summation of the components of the vector quantities inside the bracket. $\operatorname{Im}[\cdot]$ refers to the imaginary part of a complex quantity. The first term depends on the non-uniformity in the electric field strength, and the second term depends on the non-uniformity in the phase of the electric field which is the driving force for the traveling-wave DEP (twDEP) applications. In the case of series of planar electrodes patterned at the bottom substrate of a LOC device which are excited with different phases, the first term leads to levitation of particles with n-DEP response, and the second term leads to an axial motion of the particles over the electrodes. Direction of the axial motion depends on the sign of the imaginary part of the CM.

DEP force depends on the particle size, dielectric properties of the particle and the medium. Moreover, in the case of an AC field, DEP force (through CM factor) also becomes function of the frequency of the AC field. Depending on the dielectric properties of the medium and particle, DEP response of a particle can be switched from nDEP to pDEP. The frequency at which this transition occurs (i.e. the frequency at which DEP force becomes zero) is called the cross-over frequency. Actually, there may exist multiple cross-over frequencies for bio-particles [4]. Since DEP depends on the bio-particles' intrinsic electrical properties, EK manipulation techniques do not require any labeling, and are label-free. Dielectric properties of a bio-particle depend on the morphology and chemical composition of the internal matrix of the bio-particle. Therefore, each bio-particle has its own dielectric signature [41]. This issue introduces a bio-particle specific selectivity; however, also introduces a challenge. Since the biological basis of the dielectric signature of the bio-particles is not well-known, the prediction of the dielectrophoretic motion of the bio-particles in an electric field is not straightforward. For EK manipulation, both negative and positive forces can be generated with different configuration of the electrodes and/or the microfluidic channel structures, and switching the polarity and/or the frequency of the electric field. In addition to that, DEP has a favorable scaling effect which makes it perfect candidate for the manipulation of micro/nano-sized particles [8]. One requirement for the DEP force to be induced is the non-uniform electric field generated within the microfluidic device. Non-uniform electric field can either be generated by means of (i) insulator structures or (ii) by specially designed microelectrode arrays.

\subsubsection{Insulator-based DEP (iDEP)}

The non-uniform electric field can be generated by means of the specially designed microchannel network (such as serpentine channel and spiral channel) or specially designed structures inside the microchannel network (such as electrically insulated hurdles and obstacles). Typically, the electric field is applied by using external electrodes that are submerged into the reservoirs, and the flow is also induced by the electric field (i.e. electro-osmotic flow). High electric voltage is required to generate the sufficient electrokinetic force within the micro channel network for these applications which makes the use of DC field (or DC-biased AC) feasible. Therefore, commonly iDEP applications are DC-DEP applications. However, special care is needed since the high electrical voltage may lead to a serious Joule heating effect inside the channel. This severe temperature increase inside the channel due to Joule heating may lead to a bubble formation which can severely interfere with the operation of the device [42]. On the other hand, due to the absence of the electrodes inside the device, iDEP devices are robust, chemically inert and do not require any photolithography, thin-film deposition and lift-off and/or etching for electrode fabrication.

\subsubsection{Electrode-based DEP (eDEP)}

The non-uniform electric field can be generated by means of the specially designed microelectrode arrays (i.e. interior electrodes) patterned within the microchannels. To avoid the adverse effects of DC field such as migration of charged particles towards the electrodes, AC field is applied for eDEP applications. Most of the time, embedded internal electrodes are planar (2D) (i.e. height of the electrodes are in the order of hundred nanometers), and are fabricated within the device by means of complex, time consuming and relatively expensive manufacturing techniques such as thin-film deposition, sputtering, chemical vapor deposition, etc. Moreover, fouling of the electrodes may distort the operation of the device when working with bio-particles [43]. However, with an appropriate design (i.e. closely spaced electrodes), operating voltage can be lowered (which prevents any suffering from Joule heating) for eDEP applications. Moreover, low voltages simplify the equipment and circuitry. For eDEP applications, the interfacial effects may occur at the interface between the fluid medium and the electrode surface, and may lead to adverse effects such as electrode polarization, local heating around the electrodes (which may result in AC electroconvection), bubble formation and dissolution of the electrodes [8]. Therefore, some constraints need to be considered in the design of such systems.

\subsection{Acoustic-based (ACT)}

The use of ultrasonic standing waves for bio-particle manipulation relies on the creation of ultrasonic standing waves within a channel. The generation of acoustic radiation force has been studied for a long time. The formulation for acoustic radiation force on inelastic [44] and elastic spheres was derived [45] and later generalized by Gorkov [46]. In general, equations of acoustics consider the compressible Navier-Stokes equation and the first order harmonic variations on the static mean of acoustic properties. However, the time-average values of these variables lead to zero for harmonic inputs. Realistically, having zero mean for the acoustic variables over one cycle cannot be the case since from the tests it is known that there is net displacement of particles over time which means that the average of the acoustic force over one cycle cannot be zero. Therefore, for the calculation of acoustic radiation force time averaged second order radiation forces need to be used [47]. The gradient of the acoustic radiation potential can be related to the acoustic radiation force as:

$\mathbf{F}_{\text {rad }}=-\nabla U_{\text {rad }}$,

where $U_{\text {rad }}$ is the radiation potential. Under the assumptions of particles being small with respect to the wavelength of the acoustic waves and not considering acoustic wave scattering from other particles (i.e. small particles with low concentration), the acoustic radiation force can be obtained as:

$U_{\text {rad }}=\frac{4 \pi}{3} R^{3}\left[f_{1} \frac{1}{2 \rho_{f} c_{f}^{2}}\left\langle p_{i n}^{2}\right\rangle-f_{2} \frac{3}{4} \rho_{f}\left\langle\mathbf{v}_{i n}^{2}\right\rangle\right]$,

$f_{1}=1-\frac{\rho_{f} c_{f}^{2}}{\rho_{p} c_{p}^{2}}, \quad f_{2}=\frac{2\left(\rho_{p}-\rho_{f}\right)}{2 \rho_{p}+\rho_{f}}$.

Eq. (12) contains the variables related to the acoustic properties of the fluid medium as well as the particles to be manipulated. $p_{\text {in }}$ and $\mathbf{v}_{\text {in }}$ are the incident acoustic pressure and acoustic particle velocity, and $\rho_{f}$ and $c_{f}$ indicates the density and the speed of sound of the suspension fluid, respectively. $\rho_{p}$ and $c_{p}$ indicates the density and the speed of sound of particles, $R$ is the radius of the particle. The above expressions are true for any acoustic field. For the case of an acoustic standing wave where acoustic pressure is maximum, the particle velocity is minimum at the channel walls. The following 


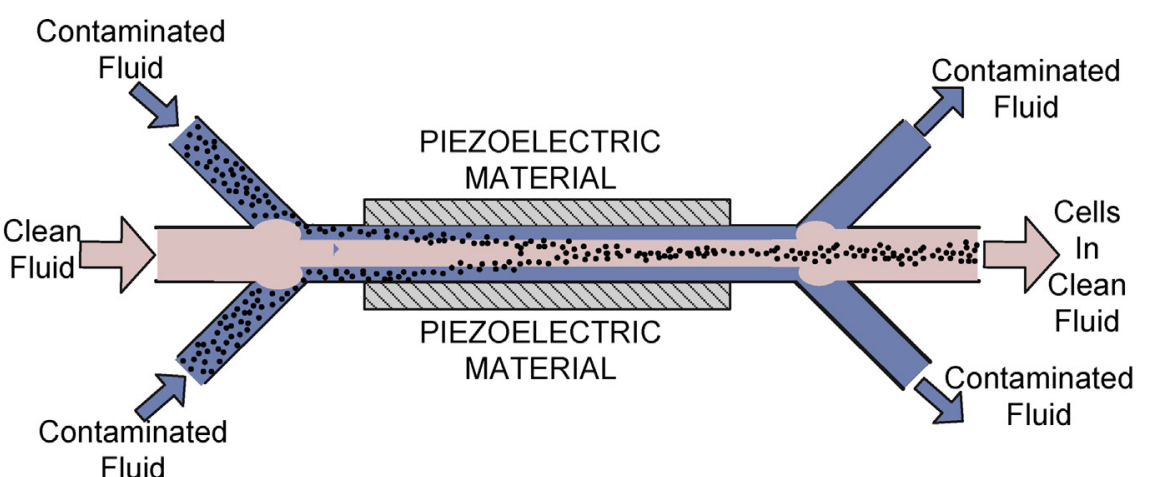

(a)

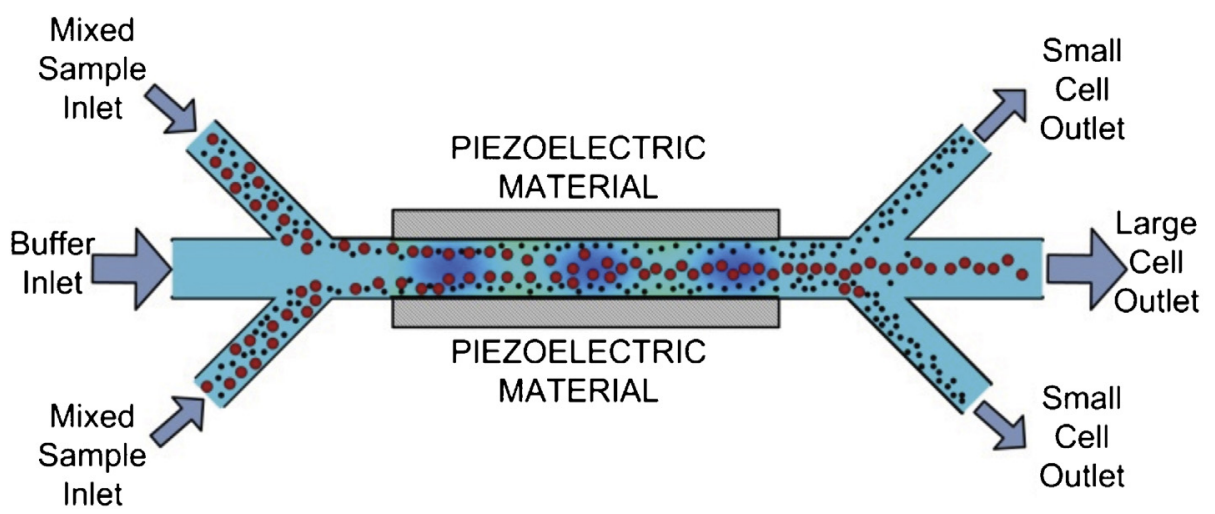

(b)

Fig. 2. A representation of acoustic based bio-particle (a) washing and (b) separation.

equation shows the acoustic radiation force due to a 1D standing wave as follows:

$\mathbf{F}_{\text {rad }}=4 \pi R^{2}(k R) E_{a c} \Phi \sin (2 k y) \hat{j}$,

where $k$ is the wave number, $y$ is the location direction along which the acoustic pressure wave changes, and $\phi$ is the acoustophoretic contrast factor which creates different amount of forcing on particles due to their acoustic properties

$\Phi=\frac{\rho_{p}+2 / 3\left(\rho_{p}-\rho_{f}\right)}{2 \rho_{p}-\rho_{f}}-\frac{\rho_{f} c_{f}^{2}}{3 \rho_{p} c_{p}^{2}}, \quad E_{a c}=\frac{1}{4} \rho_{f} u_{y}^{2}$.

The sign of Eq. (14) also determines the direction of the particles' motion when they are pushed by the acoustic radiation force. If the contrast factor is positive, the particles will move towards the nodal points and if the contrast factor is negative, particles move to the antinodes of the acoustic pressure waves. Hence, Eqs. (13) and (14) can be used to determine the acoustic radiation force applied on a bio-particle solution with low concentration such that the acoustic pressure field is not distorted due to existence of the particles. Moreover, the derivation also assumes that the radius of each particle is small compared to the acoustic wavelength.

The acoustic standing wave field creates an acoustic radiation force on bio-particles whose magnitude depends on the size and acoustic properties of the bio-particle. The difference in the magnitude of the acoustic radiation force causes the particles to be manipulated into the different locations within the microchannel based on their properties. Property dependent particle manipulation can be exploited for different applications in biotechnology. Bio-particle washing is one of these applications in which deflection of bio-particles is used to move the bio-particles from one carrying medium to another. For this to occur, there need to be two different types of carrying medium (buffer) flowing in the microchannel and the Reynolds number of the flow needs to be low to satisfy minimal mixing of the two buffer solutions. The idea behind the bio-particle washing is to move the particles from one buffer to another through the use of acoustic radiation force induced by the ultrasonic standing waves inside the microfluidic channel. The process can be seen in Fig. 2(a). Ultrasonic waves can also be utilized to trap bio-particles (acoustic trapping) to certain locations (nodal planes) of a microchannel. The acoustic radiation force may be used to overcome the drag force on the bio-particles and hold them stationary (i.e. trapped particles). The same principle can be used to clean the medium from bio-particles or increase the number concentration of the bio-particles in the medium. Since the magnitude of the acoustic radiation force induced on a bio-particle depends on the size and acoustic properties of the bio-particle, standing ultrasonic waves can be used in bio-particle separation, which is also known as acoustophoresis. Bio-particles can be manipulated to arrive at certain locations inside the microchannel at different instants. For instance, when bio-particles are directed to the center of the channel by the acoustic radiation force, the time to reach the center of the channel will be different for different bio-particles, and this difference in time can enable the separation of bio-particles. If separation in terms of size is desired, then the largest diameter particles will reach the center channel first where they can be channeled out from the center, whereas the smaller diameter particles can be channeled out from locations that are closer to the channel sidewalls. This process is schematically shown in Fig. 2(b).

\subsection{Magnetic-based (MG)}

Magnetic field can be used as an external force to manipulate bio-particles. The force applied by the magnetic field on a particle can be written as [48]:

$\mathbf{F}_{M G}=V \frac{\chi_{p}-\chi_{m}}{\mu_{0}}(\mathbf{B} \cdot \nabla) \mathbf{B}$, 


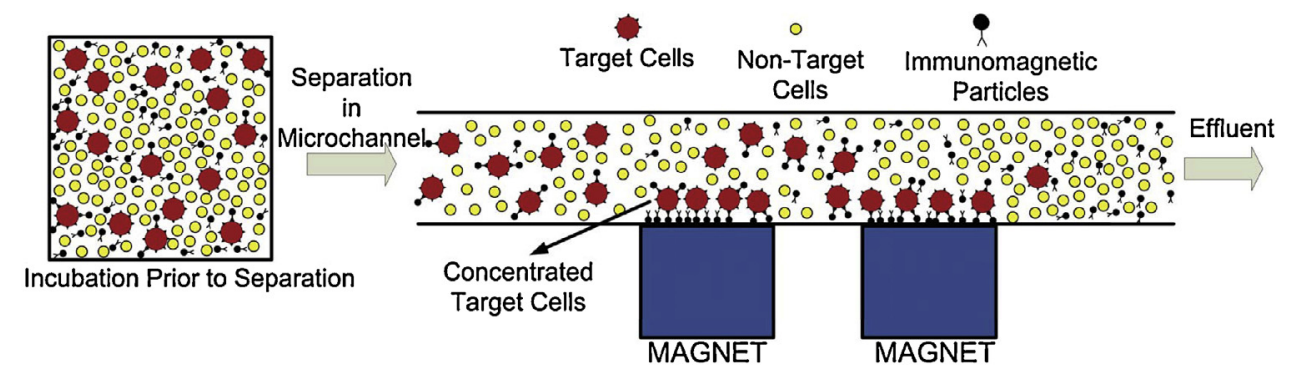

Fig. 3. The principle steps of immunomagnetic bio-particle separation.

where $\chi_{p}$ represents magnetic susceptibility of the particle, $\chi_{m}$ is the magnetic susceptibility of the medium, $\mu_{0}$ is the magnetic permeability of the free space, $V$ is the volume of the particle, and $\mathbf{B}$ is the magnetic flux density. The important parameters which affect the magnetic force on a particle are the difference between the magnetic susceptibilities of the particle and the medium, as well as the magnitude and the gradient of the magnetic flux. Moreover, the magnetic force depends on the volume of the particle (i.e. the magnitude of the force has size dependence).

Typically, it is not possible to generate large enough magnetic force on a bio-particle suspending in a conventional aqueous solution. To be able to create large enough force, the difference between the magnetic susceptibilities of the particle and the medium needs to be large. This difference may be realized either by increasing the magnetic susceptibility of bio-particles or the suspension medium. It is difficult to increase the inherent magnetic susceptibility of bio-particles; however, an engineered paramagnetic micro-particle with high magnetic susceptibility can be attached to bio-particles which causes bio-particles to be manipulated by the paramagnetic micro-particle. For the binding process to occur, the surface of the paramagnetic micro-particle is covered with antibodies which have affinity to bind onto the bio-particle (see Fig. 3). The magnetic manipulation using this process is called as immunomagnetic bio-particle manipulation. Alternatively, a ferrofluid or a paramagnetic suspension rather than a typical suspension medium such as saline or PBS (phosphate buffered saline) can be used to generate a large difference in magnetic susceptibilities of the suspension fluid and bio-particle. The use of such suspension medium eliminates the need for attaching a magnetic micro-particles onto the bio-particles. Once the field is introduced, the bio-particles can be pushed away from the magnetic field whereas in the immunomagnetic case the paramagnetic particles (hence the binded bio-particles) are attracted to the magnetic field. The repulsion of bio-particles is due to sign change in Eq. (15) since the magnetic susceptibility of the medium becomes larger than the particles. The magnetic manipulation in which the bio-particles are suspended in a paramagnetic or ferrofluid suspension medium is also called diamagnetic bio-particle manipulation. The diamagnetic cell manipulation is commonly used for focusing purposes. However, the diamagnetic cell manipulation approach can also be used for concentration and separation of particles of different sizes as illustrated in Fig. 4.

\subsection{Optic-based (OP)}

The use of radiation pressure of light to displace and trap micron-sized dielectric particles was first hypothesized and demonstrated in 1970s by Ashkin [49]. It was not until 1980s that the practical applications of optical forces in physics [50] and biology [51] have shown its true potential. The scientific community has since been using the term optical tweezers to identify the use of optical forces as a means to manipulate nanometer to micrometersized objects. Some of the significant achievements using optical traps are studying molecular motors with subnanometer resolution [52], fundamental properties of colloids and interface science [53], mechanical properties of living cells [54], and polymer elasticity [55]. The ability to precisely control small objects with no mechanical contact is definitely advantageous for microfluidic applications. Recent advances on microfluidic device fabrication brought a different perspective to the utilization of optical forces. Practical applications such as sorting, separation, and self-assembly are now possible using light-matter interaction and basic principles of microfluidics.

The basic instrumentation behind an optical trap is a high numerical aperture (NA) microscope objective that is able to tightly focus a light beam. The dielectric particle near the focus, with higher refractive index compared to its surroundings, receives the incident photons, which are scattered and/or absorbed. Scattering of incident photons creates a momentum transfer, hence the optical force component. The physical principle behind optical traps can be explained by the balance of two forces: (i) scattering forces in

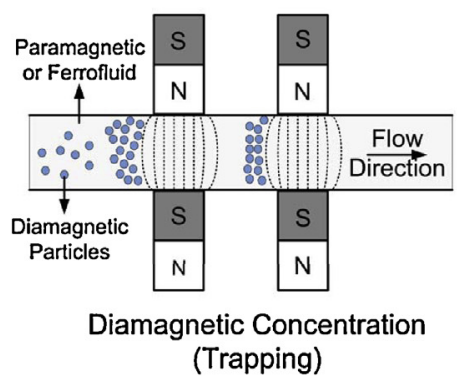

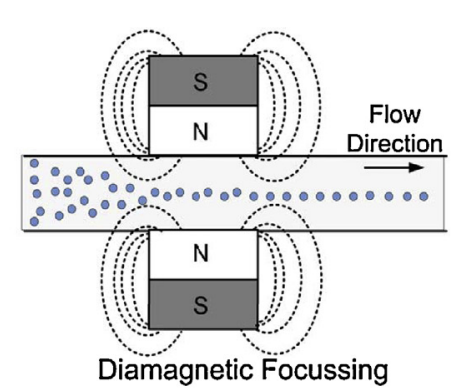

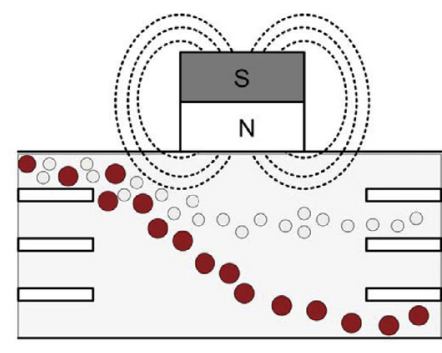

Diamagnetic Separation

Fig. 4. Diamagnetic bio-particle manipulation types. 


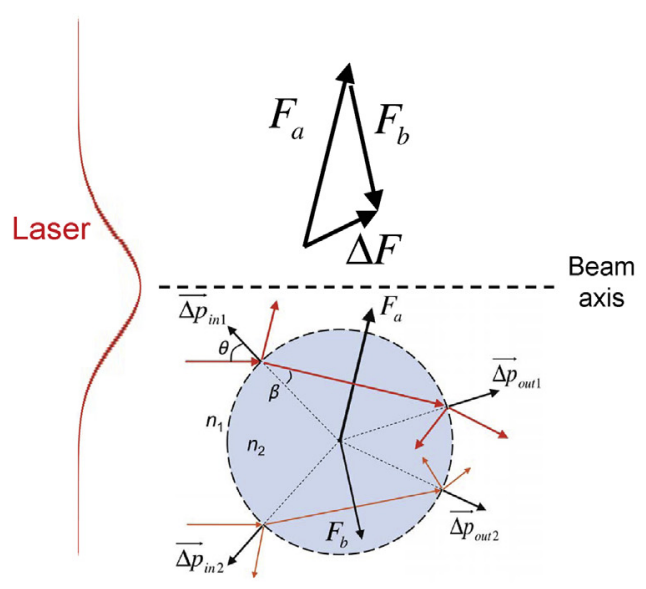

(a)

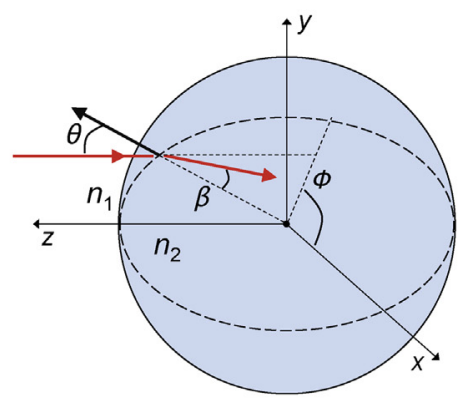

(b)

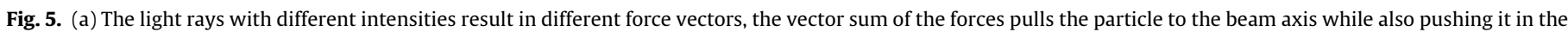
direction of beam propagation. (b) The ray parallel to $z$-axis hits the intersection plane with an angle $\phi$ with respect to $x-z$ plane.

the direction of propagation and (ii) gradient forces in the direction of optical field gradient. Scattering force generates radiation pressure that pushes the dielectric particle away from the light source. Gradient force, on the other hand, acts the opposite way and attracts the particle towards the peak spatial light intensity. In the case of tightly focused beam, stable trapping occurs if the gradient force exceeds the scattering force. Trapping depends on factors like particle size, refractive index, laser power and spatial characteristics. Theoretical treatment of optical trapping categorizes the forces on particles depending on their sizes. The condition where the size of the particle is much larger than the wavelength of light $(d \gg \lambda)$ is called the Mie regime. In this regime, the forces acting on a particle can be computed by Ray optics principles, i.e. reflection and refraction. Reflection and refraction on the front and back surfaces creates perpendicular momentum change on a sphere as see in Fig. 5(a). In the figure, a dashed circle represents cross section of the sphere and was chosen for demonstration purposes.

Assuming a Gaussian intensity profile, the ray closer to beam axis creates more point force than the ray situated further away. The net force pulls the object towards the beam axis, and pushes the object due to scattering. Gradient force only dominates and pulls the object when there is a steep field gradient achieved by tightly focusing the beam. In Mie regime, the force acting on a sphere at an incidence angle of $\theta$ and refraction angle of $\beta$ is due to a single ray of power $\mathrm{P}$ is $\left(n_{1} Q P / c\right)$. The refractive index of the medium surrounding the particle is $n_{1}$ and the particle refractive index is $n_{2}$. $Q$ describes the amount of momentum transfer at the front and back surfaces for multiple bounces, and is a function of Fresnel reflection $(R)$ and transmission $(T)$ coefficients. $Q$ value for all the rays acting on a circle were given by Ashkin [56], and the total scattering and gradient forces acting on a dielectric particle were given by Roosen and coworkers [57]. The circle on Fig. 5(a) can be treated as the intersection of a plane and a sphere on Fig. 5(b), and the total scattering and gradient forces can be integrated over every point on the sphere as:

$$
\begin{aligned}
F_{S}= & -\int_{0}^{\pi / 2} d \theta \int_{0}^{2 \pi} d \phi\left(\frac{\mathbf{E}^{2} r^{2}}{\mu_{o} c^{2}} \sin \theta \cos \theta\right) \\
& \times\left(R \cos 2 \theta+1-T^{2} \frac{\cos 2(\theta-\beta)+R \cos 2 \theta}{1+R^{2}+2 R \cos 2 \beta}\right),
\end{aligned}
$$

$$
\begin{aligned}
F_{g}= & -\int_{0}^{\pi / 2} d \theta \int_{0}^{2 \pi} d \phi\left(\frac{\mathbf{E}^{2} r^{2}}{\mu_{o} c^{2}} \sin \theta \cos \theta \sin \phi\right) \\
& \times\left(R \cos 2 \theta-T^{2} \frac{\sin 2(\theta-\beta)+R \sin 2 \theta}{1+R^{2}+2 R \cos 2 \beta}\right),
\end{aligned}
$$

where $\mu_{o}$ is the free space permittivity, $c$ is the speed of light, $\mathbf{E}$ is the incident electric field and includes the optical beam information such as its radius, $r$ is the radius of the sphere, and $\phi$ is the angle between the plane that the ray propagates and the $x-y$ plane.

The condition $(d \ll \lambda)$ is called the Rayleigh regime, describing the diffraction-limited conditions. In this regime, particles are treated as point dipoles, and the gradient and scattering forces are proportional to the real part and imaginary part of the polarizability of the particle, respectively. Scattering force, which is in the direction of incident power and the gradient force, which is in the direction of intensity gradient can be written as [58]:

$F_{S}=\frac{I_{o} n_{1}}{c} \frac{128 \pi^{5} r^{6}}{3 \lambda^{4}}\left(\frac{m^{2}-1}{m^{2}+2}\right)^{2}$,

$F_{g}=-\frac{n_{1}^{3} r^{3}}{2}\left(\frac{m^{2}-1}{m^{2}+2}\right) \nabla \mathbf{E}^{2}$,

where $m$ is the index contrast ratio, $n_{2} / n_{1}$, and $I_{0}$ is the intensity of light. The scattering force is dependent on the light intensity, the particle size, the wavelength of the light, and refractive index contrast. The gradient force is dependent on the spatial variation of electric field, the particle size and the refractive index. Unlike scattering, gradient force scales with the third power of particle size. Hence, it is easier to achieve trapping for a smaller particle than a large particle.

Since the optical trapping theory is size and geometry dependent, researchers depend on empirical determination of optical forces. The procedure is called optical force calibration (standard methods with their advantages and disadvantages can be found elsewhere [13]). The main objective of the optical force calibration is to extract the trap stiffness $(k)$ of the optical trap that is treated as a Hookean spring where the force is linearly proportional to displacement $(F=-k x)$. One common approach is the drag force calibration where the particle is held stationary using the trap, and the chamber containing the fluid is moved at a velocity so that the particle escapes from the trap. The velocity information can then be used to calculate the drag force, which is corrected based on 
the distance to the closest surface. Alternatively, Brownian motion calibration can also be used. In this approach, the fluctuations in the position of the particle are recorded, and a power spectrum is formed and fitted to extract the trap stiffness. This method also requires knowing the drag on the particle. In both methods, accurate measurements of displacement are crucial for the calculation of applied force and either a quadrant photodetector or video tracking is necessary.

\section{Assessment of the methods}

\subsection{Sample preparation and selectivity}

The selectivity of the HD methods is based on bio-particle size and deformability. If there is an appreciable size difference among bio-particles, they can be manipulated to different locations within the microfluidic network. One typical aspect of bio-particles is that they do not possess specific size, instead they posses a size distribution. If there is a size overlap for two different bio-particle populations, the selectivity of the HD methods diminishes. One major advantage of HD methods is that they do not need any labeling. Although labeling is not necessary, sample preparation may still be needed, since the concentration of the particles may affect the flow field, and result in a undesired flow patterns. For example in the case of whole blood, dilution of the whole blood may be useful depending on the application.

The selectivity of the EK applications rely on the dielectric properties of bio-particles. The dielectric state of a bio-particle depends on physical (size, shape, surface morphology) and chemical state of the bio-particle. For DC-DEP applications, DEP response can be predicted easily since it only depends on the conductivity of the bio-particle and the medium. For AC-DEP applications, DEP response also depends on permittivity of the medium and the bioparticle. Moreover, DEP response is also a function of frequency of the AC-field. To manipulate bio-particles effectively, the DEP response needs to be pre-determined. Although there are many studies regarding DEP-based manipulation of bio-particles, it is still challenging to predict the DEP response of the bio-particle of interest. The best way is to measure the DEP response of the target bio-particles. There are some proposed methods in the literature for the measurement of DEP response [8,59,60], however the process is not straightforward. A stand-alone microfluidic platform with some special circuitry and/or optical detection system is needed to determine the DEP response of a bio-particle population. If this challenge is handled, the selectivity of DEP can be utilized to manipulate different bio-particles.

The presence of the electric field in EK applications induces Joule heating within the fluid. Depending on the conductivity of the buffer solution and the strength of the electric field, the Joule heating effect can be severe, and cause deterioration of the system performance $[8,42]$. Moreover, Joule heating may also cause undesired electrothermal effects which may affect the flow field within the micro channel [8]. Since the physiological buffer solutions for bio-particles typically have high electrical conductivity, dilution of the buffer solution may be necessary for EK applications. This issue is more critical for iDEP applications in which DC electric field is present throughout the device.

For ACT applications, no special sample preparation is needed. The buffer fluid can be any kind of buffer or saline since almost all have similar acoustic properties. Particles with high contrast factors can be manipulated more effectively. A sign or a significant value difference in acoustic contrast factor of bio-particles are desirable for effective differentiation of bio-particles. When the acoustic properties and sizes of particles that need to be separated are close to each other, acoustic properties of the suspension medium can be adjusted such that the acoustic contrast factor shown in Eq. (14) is positive for one type of particles and negative for the other type. Utilizing this idea, red blood cells (RBCs) and platelets have been separated successfully [61]. Although, no bio-particle preparation (other than dilution) and labeling is necessary for acoustic manipulation, labeling of particles were utilized to have better selectivity in a very limited number of studies where bio-particles were tagged with beads which have negative acoustic contrast factors [62]. This way when the acoustic field was employed, the labeled bio-particles can move in the opposite direction of the remaining particles. Moreover, the bio-particle may be labeled with fluorescent dye to improve the visibility of the particles movement [63].

Considering the MG applications, both the diamagnetic and immunomagnetic manipulation methods require sample preparation prior to the bio-particle manipulation. In immunomagnetic bio-particle manipulation, the particles need to incubated with the bio-particle sample so that the target cells are labeled. The labeling occurs due to biochemical reactions between the antigens and antibodies on the bio-particles and the magnetic particles. The duration of the incubation is in between 10 and $30 \mathrm{~min}$ depending on the mixing, temperature and bead-cell concentrations. Moreover, if the manipulated bio-particles are to be infused back to the host, the magnetic particles need to be washed away from the selected bio-particles. However, the sample preparation step allows the immunomagnetic process to be highly selective. It is possible to separate targeted cells which do not have significant physical differences from other cell groups even if the number of total to target cell ratio is in the order of $10^{9}$ [64]. For the case of a diamagnetic particle separation, the bio-particles need to be suspended in a paramagnetic fluid or ferro fluid. The selectivity of this method can be extended by switching the magnetic field using an electro-magnet [65]. Common choices for paramagnetic fluids are $\mathrm{MnCl}_{2}$ (Managnese (II) Chloride), $\mathrm{GdCl}_{3}$ (Gadolonium (III) Chloride) and Gd-DTPA (gadolinium(III) diethylene-triaminepentaacetic acid) which is an FDA approved MRI contrast agent. In ferro fluids, the most common choice is the use of EMG408 from Ferrrotec which is a water based product with nano-sized iron particles. At the end of the manipulation process, the paramagnetic fluid or ferrofluid may need to be washed away from the bio-particles if infusion into a live organism is to be followed. Due to the sample preparation and post processing, MG methods require relatively complex procedures. There are some examples of microscale systems which perform the incubation step in-line. One such system uses six rotating magnets [66] to mix micro-particles so that incubation with the bio-particles can occur on the microfluidic device. After tagging the bio-particles, the incubated bio-particles are sent to the microfluidic device and can be positively selected by means of a magnetic field. The process of immunomagnetic separation is generally used for separation of rare bio-particles rather than focusing large number of bio-particles since the immunomagnetic tagging of bio-particles is not economically feasible and is time consuming in large numbers.

In the case of OP applications, it is important to know the physical characteristics of the fluid and the trapped object during optical trapping experiments. Refractive index changes according to medium which the bio-particle is in and the bio-particle type. For instance, the refractive index of the cell media may change during cell culture growth, although the refractive index of individual cells typically stays the same $(\sim 1.38)$. It is beneficial to measure the mediums refractive index (usually around $\sim 1.33-1.35$ ) with a refractometer if suspended bio-particles are used. Moreover, refractive index of any bio-chemical stimuli agent introduced to microfluidic channels should be known before hand. The main advantage of OP methods is the ability to sense different colors of light emitted from different fluorophores. Broad-spectrum light detectors can be adjusted with optical filters to detect only certain 
portions of spectral window. This ability is useful in active and passive feedback systems where the amount of spectral sensitivity is the identifier for the state of a bio-particle. Fluorescence or immunofluorescence staining (labeling) of bio-particles is performed using in-vitro methods depending on the size of the dye molecule. Besides dye staining the cells, the pathogens introduced to growth media during cell preparation can also be fluorescently labeled and used as the diagnostic marker during optical manipulation [67]. In OP applications, the channels need to be primed with certain chemicals prior to flow of bio-particles to prevent adhesion especially during an extended stop of the flow. In the case of biological cells, the cell media containing proteins, glucose, salt, lipids and other nutrients are good priming agents, and need to be applied prior to the operation. Bovine serum albumin is also an effective agent that prevents adhesion of enzymes to glass surfaces.

\subsection{Flow-rate and throughput}

For filtering applications, the particles with a larger size can be immobilized and/or particles with smaller size can flow through the desired outlet. Clogging is typical for filter applications. Moreover, the shear stress induced on the bio-particles may cause lysis. As more and more bio-particles are trapped within the microchannels, higher shear forces are induced, and higher pressures are required to maintain the desired flow rate. For other HD applications, bio-particles flow in a micro channel in a force-free manner. However, for DLD, PFF and hydrophoresis applications, the flow needs to controlled precisely, and regarding both the fabrication (for hydrophoresis the gap size of the contractions is critical and needs to be comparable with the bio-particle size) and the operational concerns, they need to be operated with relatively small flow-rates (in the order of $\mu \mathrm{L} / \mathrm{h}$ ). This leads to low throughput for many clinical applications. As an example, it has been shown that inertial effects decrease the sorting efficiency for hydrophoresis applications [26]. At this point, inertial microfluidics offers a promising perspectively. Inertial microfluidics utilizes the high Re characteristic of the flow; therefore, inherently flow-rates are high in the order of $\mathrm{ml} / \mathrm{h}$ which leads to high throughput ( $>\sim 10^{6}$ particles/min), and this makes inertial microfluidics suitable for many clinical applications. However, the prediction of the flow field and the particle motion in this regime is also challenging. The throughput of several HD application can be seen in Fig. 6. The highest throughput for HD manipulation is around $\sim 10^{11}$ particles/min, and belongs to an application in which the plasma is separated from whole blood rather than a differentiation of bio-particles.

The successful manipulation of bio-particles in EK applications depends on the balance between the drag force and the EK forces. An increase in drag force requires an increase in EK force and this indicates the use of higher electrical field strength. Since higher electric fields are not desirable due to Joule heating phenomena [42], the drag force and the flow-rate are limited for these applications. In the case of eDEP applications, the electric field is an issue only within the vicinity of the electrodes; therefore, higher electric fields can be tolerated. However, one major disadvantage of the eDEP devices with planar electrodes is that the electric field gradient decreases rapidly as the particles flow away from the electrodes. Therefore, an effective manipulation region is generated within the vicinity of the electrodes which reduces the effectiveness of the manipulation. If the electrodes are deposited on the bottom wall of the microfluidic channel, then the height of the channel needs to be limited. This limitation can be solved by introducing 3D electrodes within the device. Many researchers have recently realized this aspect and proposed different fabrication techniques to fabricate embedded 3D electrodes [68]. By introducing 3D electrodes, the flow-rates and the throughput of the DEP-based devices can be enhanced although throughput of EK methods is relatively low, and at this point not suitable for clinical applications. The throughput of several EK applications can be seen in Fig. 6 .

In terms of throughput, ACT devices have relatively higher throughput compared to that of other methods. The flow rates generally range from $1 \mu \mathrm{l} / \mathrm{min}$ to $1 \mathrm{~L} / \mathrm{h}$. The throughput of ACT devices for some studies can be seen in Fig. 6. The figure shows eight orders of magnitude difference in the bio-particle throughputs. The studies with high throughputs are generally studies which process whole blood samples. The normal blood count of a male is approximately $5 \times 10^{9} \mathrm{RBCs}$ in a milliliter of blood. For the applications which do not include fractionation of different cell groups from each other such as separation of plasma from whole blood, the throughput reaches as high as $10^{11}$ particles/min [69].

Throughput of MG manipulation methods is also promising. The throughput of several MG applications can be seen in Fig. 6. The largest of the two throughput values $[70,71]$ belong to a system where the bore diameter of the channel is roughly $8 \mathrm{~mm}$. It may be argued that this diameter is too large for a microfluidic system. Most of the higher flow rates belong to systems with immunomagnetic bio-particle manipulation systems, and diamagnetic particle manipulation systems have lower throughputs. However, it should be noted that the throughputs are purely based on the time that cells spend in the microfluidic circuit. In the immunomagnetic particle manipulation cases, a significant portion of the time is spent on the pre-processing of bio-particles (considering the incubation time for tagging of bio-particles with the magnetic particles), hence the throughputs may not be representative of the total time required for the bio-particles to be processed. Generally, flow velocities in the microchannels are in the order of $\mathrm{mm} / \mathrm{s}$ or less.

For OP applications, active microfluidic cell sorters compared to its passive counterparts use information such as fluorescence, size and shape by means of photodetectors or image processing to dynamically steer the bio-particles to desired outlets. These systems can operate quite fast based on the type of force used for manipulation. For instance, active sorters either utilize optical forces for grabbing or deflection of bio-particles. Grabbing and deflection occur when the gradient or scattering force dominates each other. Active sorters based on deflection are much faster since there is no need for steering the focused optical beam. 6354 cells/min [72] and 1320 cells/min [67] has been for deflection-based sorters. Grabbing-based sorters are almost an order of magnitude slower with reported values of 300 cells $/ \mathrm{min}$ [73] and 84 cells/min [74] (the throughput of several OP application can be seen in Fig. 6). For active microfluidic systems involving OP manipulation, the flow-rate in the channel and the drag force on the bio-particle competes against the optical force. Considering the optical force on a particle in the range of a few to tens of $\mathrm{pN}$, the fluid velocity needs to be adjusted to achieve desired manipulation for the bio-particle.

\subsection{Channel geometry}

The filtering applications in HD methods typically include a microfluidic channel with post structures. The spacing of the post structures is critical to trap the bio-particles with target size. Depending on the size of the target particles, the spacing can vary between 5 and $15 \mu \mathrm{m}$. To introduce as many post structures as possible for a given volume, the diameter (or width) of the post structures are typically around couple of tens of micrometers. For DLD applications, again some post (or pillar) structures are also needed. These post structures may have different shapes (circular, square, etc.). Since some amount of lateral displacement is desired to manipulate the bio-particles, the section with the post structures needs to have some threshold length which is typically couple of centimeters. For the PFF applications, rectangular microchannels with sharp corners are required. Although the inlet section of the 


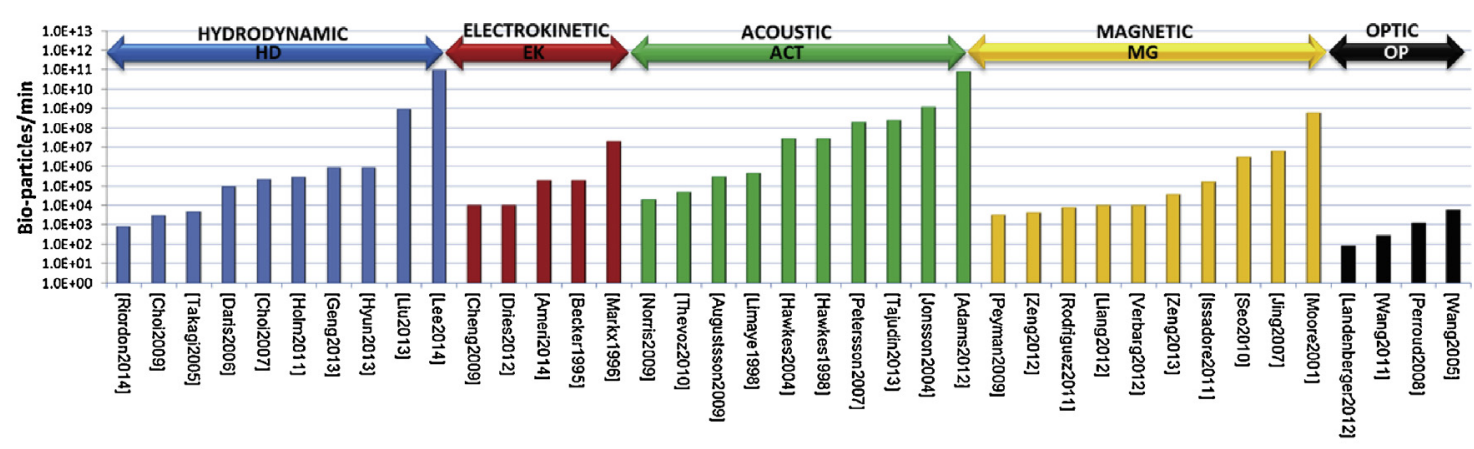

(a)

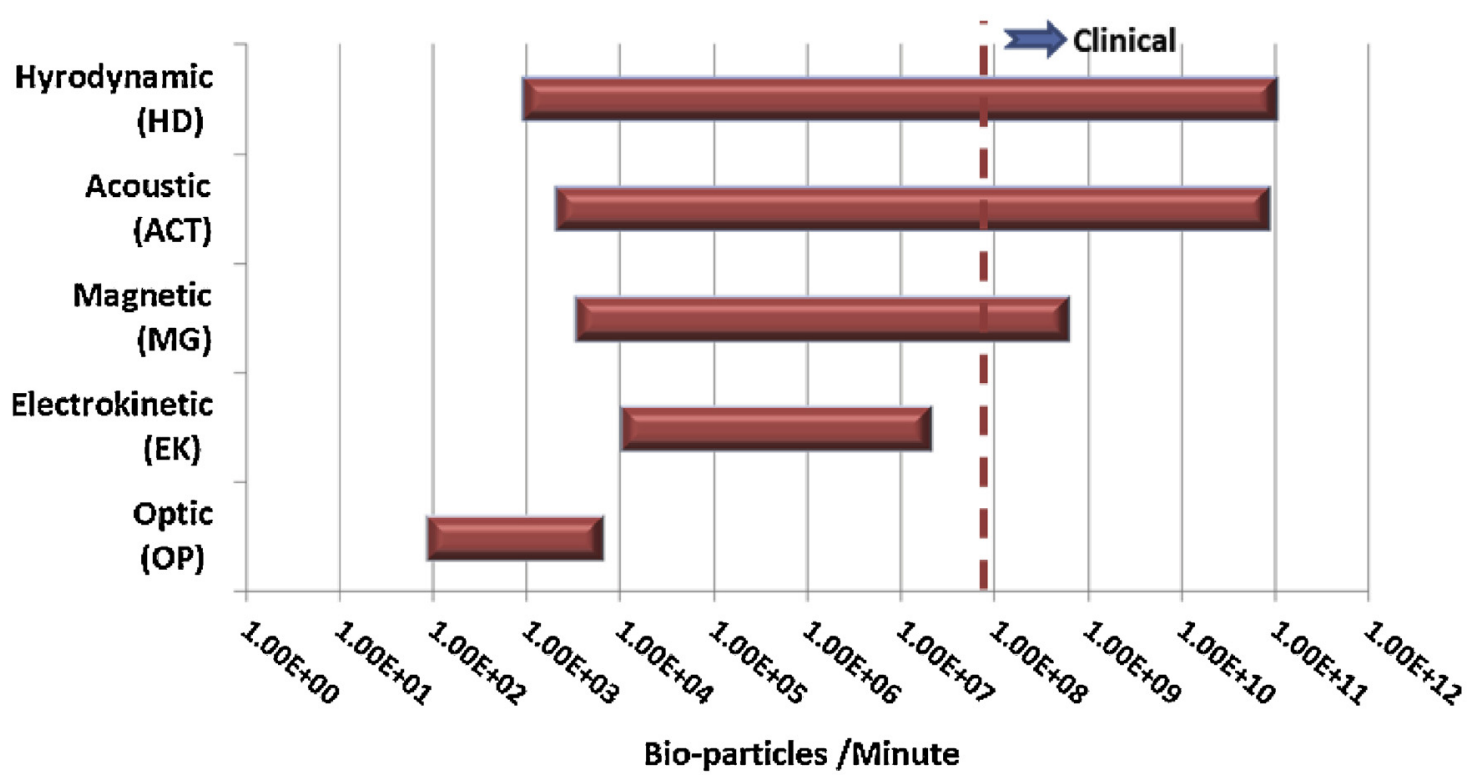

(b)

Fig. 6. (a) Throughput data for several microfluidic devices. (b) Throughput range for different methods.

device is composed of a microchannel with $\sim 100 \mu \mathrm{m}$, to achieve fractionation with a good resolution, the exit section of the device needs to be wide, and the geometry of the exit section needs to be critically designed to obtain the desired output. For the inertial applications, since the flow-rates are high to achieve high Re flows, certain length is required to achieve the required lateral displacement of bio-particles. To utilize any circulation within the flow field, it is typical to introduce an expansion/contraction sections and/or post structures within the flow field. More recently, the expansion/contraction in the height direction has also been utilized for both sorting and focusing [38]. To utilize the Dean flow for particle manipulation, spiral and serpentine channels are required, and the radius of curvature of the channels is critical [32]. For hydrophoresis applications, expansions/contractions in the height directions are necessary. The size of these contractions are critical, and certain design procedures need to be followed depending on the size of the bio-particle of interest. Typically the minimum channel height in the contracted part needs be larger than the bio-particle size to avoid clogging, and less than two times the particle size for successful manipulation. One common point about the microfluidic channel network with post structures is that the height of the channel is limited considering the limit of the aspect ratio due to the fabrication constraints.

For EK applications without internal electrodes, a typical channel width is in the order of $100 \mu \mathrm{m}$ together with some specially designed structures. The height of the microchannel is not critical as long as the fabrication is not problematic. For eDEP applications with internal electrodes, the height is critical if 3D electrodes are not used. Typically, channel height varies between 20 and $50 \mu \mathrm{m}$. For eDEP applications with 3D electrodes, microchannel structures with larger height is possible (typically 20-100 $\mu \mathrm{m}$ ). In these applications, the limitations for the height comes from the fabrication step of the electrodes.

Channel geometry is quite important for successful implementation of ACT methods. The channels generally have rectangular cross sections. It is significantly easier to couple a piezoelectric patch to a rectangular surface compared to a circular one. The width of the channel (width is the primary direction along which acoustic radiation force acts on bio-particles) needs to be carefully selected as an integer multiple of half wavelength of the acoustic waves within the fluid for the selected frequency. If the transversal configuration is selected for the piezoelectric material placement (piezoelectric materials surface not aligned with the acoustic radiation force direction), the channel width also needs to be selected as the half wavelength of the acoustic waves in the suspension fluid. However, if the layered configuration is preferred, then the channel width can be several wavelengths of the acoustic waves in the suspension medium. Not only the channel width, but also the width of the device needs to be selected as an integer multiple of the acoustic wavelength. The depth of the channel is generally selected significantly less than the acoustic wavelength in the fluid to avoid fitting a standing wave along its direction. The length of the channel is 
generally in the order of several centimeters which can fit several wavelengths. Since the acoustic waves are required to fit both into the channel and the device, the sensitivity of the performance to the channel and device geometry is significantly high. Therefore, the dimensions of the ACT bio-particle manipulator devices need to be controlled precisely.

MG manipulation has very few constraints on the geometry of the channel. Unlike the ACT method, the performance of the system is not highly sensitive to channel and device geometry. Typically, the width of the microchannels is in the order of $100-700 \mu \mathrm{m}$, the depth is in the order of $10-100 \mu \mathrm{m}$, and the length of the channel is several centimeters. Although preferred geometry is a rectangular cross-sectioned microchannel, the capillary tubes can also be utilized $[75,76]$. If permanent magnets are used, generally the poles of each magnet is brought to the vicinity of the channel walls. The closer the poles are to each other, the stronger the gradient of the magnetic field is, the (B. $\nabla)$ B term in Eq. (15), which results in stronger magnetic force on the particles.

In OP applications, the channel geometry needs to be adjusted depending on the optical beam characteristics. The focused $\mathrm{TEM}_{00}$ mode beam size, depending on the microscope objective used, is smaller than most bio-particles, and does not bring any channel size limitations. However, rectangular channel geometries is preferable to avoid optical beam shape change (e.g. circular to elliptical), and to obtain better imaging. Optical clarity is strongly affected by curved channel walls. For diverging single mode Gaussian-like beams, the beam diameter and optical power both have direct impact on the optical force and stress profile. For the applications with high numerical aperture (NA) objectives, the working distance of the objective may be less than a millimeter, and an index matching gel may be needed to take advantage of high NA. The distance between the beam origin and the light-particle interaction area; hence, the channel geometry should be carefully designed both to accommodate the optical setup and to achieve desired physical effects.

\subsection{Material and fabrication}

When the fabrication of the microfluidic devices is concerned, there are basically two common approaches: direct substrate manufacturing (photolithography, etching, laser ablation etc.) and mold-based techniques (hot embossing, injection molding or soft-lithography) [77]. Photolithography has good ability to manufacture very small and complicated microchannel structures, but it usually involves multi-step processes which take considerable time and specific chemical requirements especially for etching steps in high tech facilities such as a clean-room environment. Moldbased techniques require a mold (sometimes mold is referred as the mask) to be fabricated. Although the fabrication of the mold may need lithography-based, relatively complicated fabrication process; once the mold is fabricated, the mold may well be used for several times. After the completion of the mold, the rest of the fabrication procedure is simple and highly reproducible (i.e. low-cost replication), which makes mold-based techniques very suitable for mass production. A common material used in the fabrication of the microchannels is the Polydimethylsiloxane (PDMS) due to its low cost, low toxity and transparency. Bonding PDMS with glass can be achieved using a straightforward surface treatment process with oxygen plasma (a sealed PDMS microchannel can withstand pressures up to five bars [1]). Soft-lithography using PDMS is a very common technique used in the fabrication of many microfluidic devices for different aforementioned applications.

For direct substrate manufacturing, a common approach is to etch the wafer and seal it with PDMS. Especially, for microfluidic channels with high aspect ratio post structures $(A R \sim 1-5)$, it is preferred to use etching of the silicon wafer instead of the fabrication of a mold. For low aspect ratio post structures, molding is a better choice. For direct substrate manufacturing, an alternative technique is laser ablation, which is localized, non-contact removal of the material from the surface by exposing the surface to a laser beam. Unlike photolithography, laser ablation does not require a mask and may be applied to a wide variety of substrate materials [1]. Although the cost of the process is relatively low, the investment cost of the equipment is relatively high. Moreover, generally the surface roughness of the laser ablated channels are not superior than that of mold-based techniques [78].

For the fabrication of the devices for HD applications, softlithography and photolithography is a common approach, and fused silica, PDMS, PMMA and silicon are the common materials. In some inertial [38] and hydrophoretic [27-31] applications, 3D geometries are needed. 3D geometries can be achieved by utilizing two-step lithography. If 3D structures are both on the bottom and the top wall, lithographic aligning procedure is required $[27,28]$.

For the fabrication of devices for EK applications, softlithography with PDMS is a common method. For many EK applications, embedded electrode structures are also required which are designed and located strategically within the microfluidic structure. As a common practice, the planar electrodes within the microfluidic devices are fabricated using metal deposition on a substrate. Additional fabrication steps needs to be introduced for the devices with 3D electrodes. Different fabrication strategies for DEP-based microfluidic devices have been reviewed recently by Li et al. [68]. Titanium, gold, silver and carbon are common materials for the electrodes [68]. Copper has also been used in the literature, but some adverse effects were reported [79,80].

For the fabrication of devices for ACT applications, there is a wide variety of materials used for the microfluidic device such as silicon $[61,81,69]$, steel [82-84], glass [85,86] and PDMS [87-91] as well as other polymers [92,93]. The choice of material for the transversal configuration should have high acoustic impedance such as silicon, whereas the layered configuration may be manufactured from a wide range of materials ranging from different polymers to steel. The material of choice for acoustofluidic devices, which is excited with surface acoustic waves, is polymers with low acoustic impedance and with good shear wave carrying capacity such as PDMS [87-89]. For the silicon-based devices, fabrication through standard photolithography and wet or dry etching are common approaches [61,94,69,95,47,96]. In the case of polymers and particularly PDMS devices, standard soft lithography methods [87,89,93] and rapid prototyping are commonly employed [97]. Typically, a transparent material with higher acoustic impedance such as glass or fused silica is used as a lid to form the channel. This lid is also utilized as an acoustic reflector in transversal configurations.

For the fabrication of devices for MG applications, there is no significant constraint other than material of the device not being a magnetic material. Due to the ease of process and possibility of embedding the magnets into the device, most widely used material is PDMS [48,64,98,99,65,100-102]. Other materials are also used in this method such as PMMA [66] and silica [76]. The use of NDFeB magnetic powder together with PDMS can form self-assembled magnetic configuration and achieve better device performance for capturing applications [99].

For OP applications, microfluidic channels with optically transparent walls are required. Most transparent materials such as glass, fused silica, PDMS, PMMA are suitable owing to their transparency to visible and near-infrared spectrum. Silicon is reflective to light above its bandgap (below $\sim 1100 \mathrm{~nm}$ ), and the trapping laser should emit at optical wavelengths above this limit in the case of silicon as the trapping surface. As noted in the next section, biological viability is reduced at higher wavelengths due to increased water absorption. Thus, materials transparent to visible light are preferred over silicon for bio-particle manipulation. For the fabrication of 


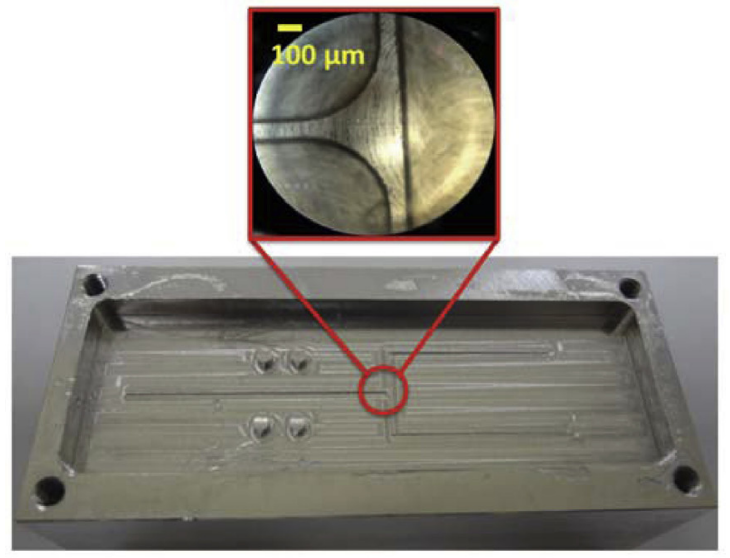

(a)

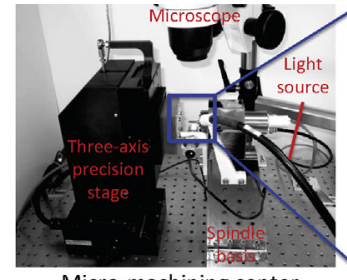

Micro-machining center

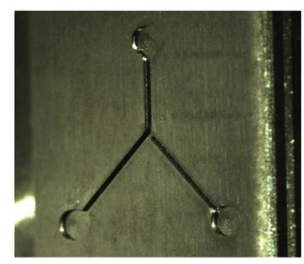

Brass mold

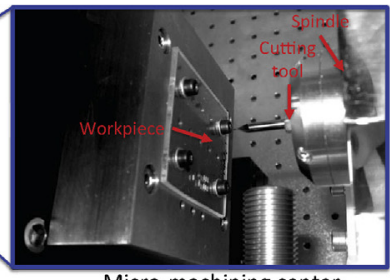

Micro-machining center

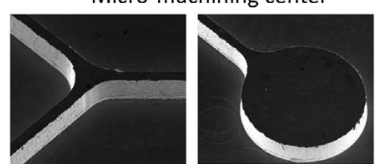

SEM image of the mold

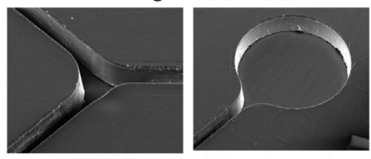

SEM image of the channels

(b)

Fig. 7. (a) Photograph of the mold fabricated with conventional CNC-machine. (b) Photograph of the micro-machining center, the mold and the channels.

devices for OP applications, standard aforementioned microfabrication techniques can be applied. Well-known processes such as soft lithography to fabricate PDMS and wet or dry etching to fabricate glass channels are popular among other techniques due to ease of use. Alternatively, 3D laser micromachining and hot embossing are also good candidates to form rigid polymeric channels.

One alternative method to fabricate the microfluidic device is to use mechanical micromachining (i.e. CNC-machining) either for direct substrate manufacturing or for the fabrication of the mold. For direct substrate manufacturing, the limits of the process is constrained by the size of the milling tool which may lead to unsatisfactory end-product for microfluidic applications. However, for the fabrication of the mold, the process is limited by the xyz-accuracy of the tool-positioner of a CNC-machine since the negative of the microfluidic structure is fabricated as the mold. With today's technology, by using magnetic bearings for their positioning systems, the $x y z$-accuracy of a conventional CNCmachines are around $5 \mu \mathrm{m}$. Therefore, a mold can be fabricated using mechanical machining within couple of hours without any need for clean-room equipment within the desirable accuracy limits for microfluidic devices. Moreover, CNC-machining can generate 3D structures without any difficulty. Common mold materials for mold-based techniques are silicon (quartz/glass), SU-8 photoresist, polymer based materials (e.g. plexiglas) or any metal based materials (titanium, stainless steel, etc.). Polymer- and metal-based mold materials are superior over silicon or photo-resist based mold materials in terms of durability and robustness. In the case of mechanical micro-machining, any of these materials can be selected. Machinability, cost and the expected life-span of the mold are the important parameters which need to be considered during the selection of the mold material. Another important parameter is the expected life of the mold. Considering the use of the mold to produce more than 7,000-10,0000 parts, metal-based materials are the best choice. However, using metal-based materials comes with a price. Machining of metal-based materials is costly due to the reduced tool life and increased machining time. On the other hand, machining of polymer based materials is less problematic in terms of tool life and machining time, yet the mold still can be used for many times.

The accuracy of the CNC-machining can be further improved by the introduction of specially designed micro-machining centers. These micro-machining centers can operate with an improved spindle speed which leads to a superior surface finish with an improved xyz-accuracy. Within Bilkent University Micro System Design and Manufacturing Center, one conventional CNC machine with magnetic bearings and one custom-made micro-machining center is available and used for the fabrication of the molds of the various microfluidic devices [103,104]. An example mold fabricated with conventional CNC-machine, and a mold fabricated with the micro-machining center can be seen in Fig. 7. The fabrication of metal electrodes with CNC machining is also possible which leads to 3D metal electrodes. An initial attempt has been made for the fabrication of an eDEP device with 3D electrodes. A microchannel with a width of $100 \mu \mathrm{m}$ and a height of $100 \mu \mathrm{m}$ was fabricated. The device and the mold can be seen in Fig. 8. Total machining time for the mold and the electrodes took approximately $180 \mathrm{~min}$. The embedded electrodes were also removed from the device and reused for the fabrication of a duplicate device without any problems.

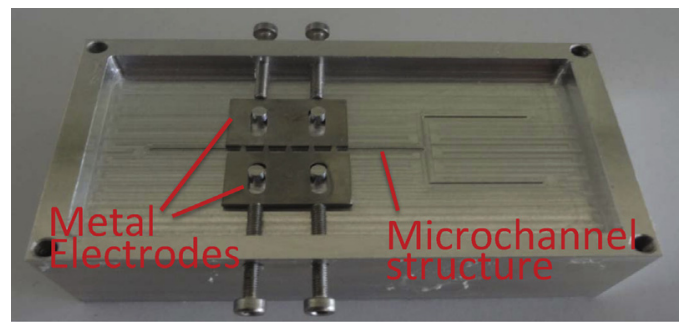

(a)

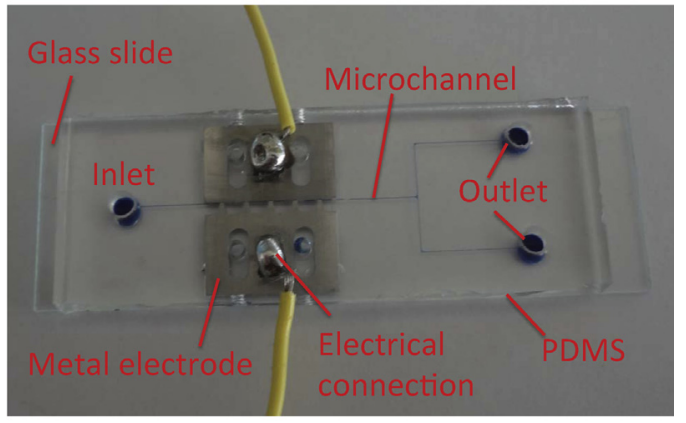

(b)

Fig. 8. The DEP-based microfluidic device with 3D electrodes fabricated by machining: (a) the mold together with the electrodes, (b) the assembled device. 
CNC-machining is rather an alternative method not a competitor for the lithography-based techniques. Especially for the mold-based fabrication, it offers unique advantages: (i) post structures with aspect ratio around 1.0 can be introduced without any difficulty, (ii) the 3D geometries with varying channel length can be fabricated easily, (iii) the angle of the microchannel sidewall on the mold and the height of the microchannel can be controlled precisely within the accuracy limit of the machining center, and deep channels can easily be fabricated. On the other hand, structures with sharp corners or an obstacle with corners is problematic since certain fillets always exist depending on the radius of the tool.

\subsection{Hardware}

For HD applications, the important parameter is the flow field. Flow field needs to be controlled through precise pumps and valves. For microfluidic applications, the use of syringe and/or peristaltic pumps are common. Due to the superior flow control and ease of use syringe pumps are more popular in microfluidic applications especially for low flow rates $(\sim 1-100 \mathrm{ml} / \mathrm{min})$. More recently, LabSmith Inc. (www.labsmith.com) has introduced micro syringe pumps (flow-rates ranging between $50 \mathrm{nl} / \mathrm{min}$ and $5 \mathrm{ml} / \mathrm{min}$ ), automated valves and microfluidic components specially designed for microfluidic applications.

For EP and iDEP applications, DC electric field is typically applied throughout the channel, and the flow is induced by electro-osmosis. Depending on the electrode geometry and flow rate, a high voltage source may be necessary. For eDEP applications, AC electric field is preferred and the applied frequency ranges from few $\mathrm{kHz}$ to tens of $\mathrm{MHz}$ with amplitudes from 5 to $100 \mathrm{~V}$ peak-to-peak depending on the electrode geometry within the microchannels. Application of DC voltages requires less auxiliary equipment (e.g. signal amplification equipment or an RF amplifier) with respect to AC although conventional function generators may be enough for most applications. Typically, using AC frequency complicates the DEP setup. Moreover, the frequency of $A C$ field needs to be tunable owing to frequency dependent CM factor. Even though it is desirable to have DEP operation with a single set frequency, especially for commercial point-of-care products, frequency tuning may be required in practice. Conventional function generators typically are equipped with such feature. The EK systems and applications include frequency dependent components with two or more electrical ports, and the electrical properties of components and materials are characterized either using LCR meters or impedance analyzers. LCR meters are usually preset and used at certain frequencies of $\mathrm{kHz}$ range. Impedance analyzers are preferred due to the ability to sweep in frequency domain from few $\mathrm{kHz}$ to $\mathrm{RF}$ range. Impedance measurements can also be applied to extract dielectric properties of bio-particles when they are nearby the induced electric potential [105]. Accurate determination of bio-particles dielectric properties is another critical requirement for commercialization of DEP devices since bio-particles of similar sizes may exhibit positive or negative DEP forces at the same operation frequency. Similar to impedance analysis, DEP affinity separation or crossover frequency determination allows measurement of frequency dependent DEP force and identification of two dielectrically dissimilar bio-particles (e.g. blood cells and cancer cells) [106]. Crossover frequency determination method involves electro-rotation and requires a swept frequency generator and electronics to enable accurate phase shifting of the generated signal.

For ACT applications, a voltage source is necessary to create the acoustic wave and acoustic radiation force. Typically, a piezoelectric patch is used as an acoustic wave source. Piezoelectric patches are ideal due to their low cost and efficient radiation at ultrasonic frequencies. The piezoelectric patch attached excites the material which carries the waves to the medium where the particles are suspended in. The use of piezoelectric ceramic material (such as PZ26) is common due to their high voltage abilities. The position of the piezoelectric material with respect to the device determines which type of configuration the resonator is in. There are three main configurations where the piezoelectric material can be placed against the chip which are transversal $[61,92,107,94]$, layered $[82,108,85,109]$ and surface acoustic wave (SAW) configurations[87-89]. In the transversal configuration, the piezoelectric material is placed on the device such that the standing wave is positioned along an axis perpendicular to the normal of the piezoelectric patch surface. The layered configuration requires the surface normal of the piezoelectric patch to be aligned with the direction along which standing waves are positioned. Surface acoustic wave configuration generates the acoustic radiation force through propagation of surface waves on the device. To actuate the piezoelectric material, a power amplifier that can operate at radio frequencies and ultrasonic frequency ranges is required. Typically, the power required for ACT applications is in the order of couple of Watts. However, in some high throughput studies with PDMS materials, it can go up to $10 \mathrm{~W}$ [97] which requires an additional external cooling of the piezoelectric material and the channel. The use of aluminum block as a heat sink may be applied $[85,97,110]$.

For MG applications, the only hardware required is a mechanism to create magnetic field gradient inside the microchannel. Generally, permanent magnets are used to create the required magnetic field, and the choice for the permanent magnet material is almost always NdFeB (Neodymium iron boron) magnets. The use of permanent magnets rather than electro-magnets simplifies the design and eliminates the need for power. On the down side, the use of permanent magnets makes it difficult to control the magnetic field strength, and it does not allow the use of switching field which may add additional selectivity to the process [65]. The pole configuration of the permanent magnet depends on the type of manipulation being performed (focusing, concentration or separation) as well as the choice of method for manipulation (diamagnetic or immunomagnetic). The strength of the magnetic field flux is generally in the order of 0.1-0.2 Tesla, but the total magnetic flux can be as high as 1 Tesla. The distance between the magnet pairs can range from tens of $\mu \mathrm{m}$ [111] to several $\mathrm{mm}$ [75]. Bio-particles can also be manipulated using the magnetic field induced by the permanent magnet on a ferromagnetic wire placed inside the microchannel $[112,113]$. Additionally, electrodes can also be introduced within the device to switch the magnetic field [65], and to provide high resolution control over positioning of bio-particles [100].

An optical tweezer setup is usually built around a microscope, and all the electronic and optical elements are integrated. Commercial optical tweezer setups are available for purchase (Thorlabs Inc., www.thorlabs.de), yet most setups are built from scratch, and customized according to the need. Handling biological specimen requires extra attention during selection of the laser due to optical damage and heating effects. Since biological specimen show low index contrast with respect to its surroundings, the amount of laser power to trap and move such an object is higher than that of a polystyrene bead. The typical laser powers range from a few milliWatts to a Watt, and absorption increases linearly with power. The choice of laser wavelength is even more critical for biological objects due to low water and protein absorption requirement. Visible spectrum (400-700 $\mathrm{nm}$ ) has the lower water, but higher protein absorption. On the contrary, wavelength spectrum above $1250 \mathrm{~nm}$ introduces many orders of magnitude higher water absorption; therefore, a heating problem that may damage the biological specimen can occur. The most preferred wavelengths for trapping are around 800 and $1064 \mathrm{~nm}$. Laser diodes at these wavelengths with optical fiber ends, not only provides high optical power, but also has a very good quality single-mode beam profile suitable for optical trapping. Traditional optical tweezers require a clean Gaussian 
$\mathrm{TEM}_{00}$ mode out of a laser, which satisfies the sharp intensity change required for high gradient force. For slow manipulation of biological objects in microfluidic channels, this requirement must also be satisfied. However, for applications that only utilize the radiation pressure to push or deflect biological objects (i.e. high speed sorting), a high-powered and focusable laser source is adequate.

\subsection{Application}

HD methods mainly use the shape and the size of the bioparticles to manipulate them; therefore, mainly HD devices have been proposed for separation and sorting of (bio)particles by size $[16-18,21-27,29-31,33-35,37-39,114,115]$ and shape $[19,18,20,28,40]$. Separation by size includes (i) enrichment of low-concentrated cancer cells from the peritoneal wash [114], separation of plasma from whole blood [115] using filtration, (ii) separation of white blood cells (WBCs) and RBCs [17], separation of plasma from whole blood [17], separation of parasites from human blood [19], capturing of circulating tumor cells (assisted by affinity-based cell culture) using DLD, (iii) separation of RBCs and WBCs [22] and separation of U937 (human leukemic monocyte lymphoma cell line) cells at different phase using hydrophoresis, (iv) enrichment of leukocyte [30] using PFF, and (v) separation of Escherichia coli from RBCs, separation of WBCs, MCF-7 and MDAMB-231 breast cancer cells [116], focusing of Jurkat and MCF7 (a breast carcinoma cell line) [38], separation of HeLa and MCF cells [34], focusing of HeLa and Jurkat cells [39], separation of plasma from whole blood [117] using inertial microfluidics. Separation by shape includes (i) separation of parasites from human blood [19], filtration of budding yeast cells [18], separation of RBCs [20] using DLD, (ii) separation of RBCs from whole blood [28] using PFF, (iii) focusing and separation of Saccharomyces cerevisiae yeast cells [40] using inertial microfluidics. More recently, an integrated microfluidic device has been proposed for real-time volume measurement and actively controlled pressure-driven flow to sort the particles by volume [118]. Sub-populations of G1-phase and M-phase of a single culture of $S$. cerevistiae yeast cells sorted at $100 \%$ efficiency. The same device was also implemented for size measurement in a non-sorting mode. The throughput of the device was reported as 30 cells/min in sorting mode, and 900 cells/min in counting mode. Although the throughput is low to process high numbers of bioparticles, superior sorting efficiency makes the proposed design an ideal device for sorting small numbers of bio-particles at very high resolution.

Typically, population of bio-particles is heterogeneous even if the population is genetically identical. Conventional methods which assess the average responses from a population may be misleading to understand the individual cell dynamics. Therefore, single cell analysis is very important. Microfluidics offers a great opportunity to analyze the inherent complexity of the cellular systems on a single cell basis with dynamic monitoring [119]. Bioparticles can be trapped in a microchannel network by introducing specially designed structures. Recently, Bell et al. [120] proposed to use of hydrodynamic traps to capture and hold Schizosaccharomyces pombe cells (fission yeast) in a well-defined array with minimal effect on growth kinetics to monitor their growth using superresolution microscopy.

EK methods (more specifically DEP) have been implemented for the separation of WBCs and breast cancer cells, separation of different bacteria species, concentration of $E$. coli and $S$. cerevisiae cells, trapping of chromosomal DNA from lysed E. coli cells, trap and concentrate single- and double-stranded DNA molecules, trapping of linear and supercoiled DNA molecules, separation of cancer cells from blood stream, isolation of malaria-infected cells from blood, separation of electroplated and non-electroporated cells to name a few [8] (an extensive review of the studies up to 2010 can be found elsewhere [7,8]). DEP has been implemented for the separation of $S$. cerevisiae and Lactobacillus casei, and L. casei and Jurkat cells [121]. In a recent study, EK methods have been utilized for trapping and lysis of RBC [122]. AC-field was utilized for trapping, and DC-field was utilized for the lysis. Moreover, trapping and lysis were monitored by measuring the impedance between the electrodes. DEP has also been used to enhance the filtering process for a sample preparation platform for depleting cells and abundance proteins from blood [123].

In biofuels production systems, the target microalgae with high lipid content is preferable to ensure high lipid productivity for a better economic feasibility. Therefore, the differentiation of lipid-rich and fast-growing microalgal species is a vital step for the development of oil-based microalgal biofuels production systems. It has been also demonstrated that DEP has also a potential to differentiate microalgal species with different lipid contents [124].

ACT methods have been implemented for bio-particle wash $[95,47,96,84,125,92]$, trapping [125,92,126-134] and separation $[69,108,85,91,9,81,135,94,136,137]$. Bio-particle washing relies on focusing the particles to the center of the channel, and out flowing the bio-particles collected at the center into another suspension medium. Utilizing this concept, the bio-particles such as RBCs $[95,47,96]$ and yeast $[84]$ have been washed with a microfluidic device. The idea of acoustics based bio-particle trapping can be used in the applications of bio-particle enrichment (concentration) [125] as well as clarification of these bio-particles from the suspension [92] through entrapment at certain locations within the microchannels or trapping them in droplets [126]. Moreover, using trapping force, patterning of bio-particles at certain locations may also be utilized for the collection of bio-particles for bio-assaying applications [92,127]. In order to generate the acoustic waves for trapping the bio-particles, confocal piezoelectric materials which are able to focus ultrasonic waves [128], as well as configurations of piezoelectric transducer in transversal and layered configurations [129] may be used. The piezoelectric material may be placed directly in contact with the fluid where particles were suspended, and particles can consequently be trapped under the generated acoustic radiation force [130]. It is also possible to increase concentration of bio-particles by directing them to the center of the microchannel, and during this movement removing the fluid from the channel which results in increased number of particles per unit volume of fluid. Implementing this idea, 200 fold increase in concentration was obtained for human cells [131]. The main limitation with ultrasonic trapping/concentration is in the use of sub-micron particles. The acoustic radiation force is not large enough to trap the particles as the size of the particle gets smaller (note that the acoustic radiation force changes with the cube of the diameter of particles). The acoustic radiation force is used to overcome the drag force, so that particles can be trapped. The fluid drag force changes linearly with the diameter at the creeping flow limit. Therefore, as the diameter of the particle gets smaller, the acoustic force reduces significantly compared to the drag force. Generally speaking, bio-particles below $1-2 \mu \mathrm{m}$ are difficult to trap using the aforementioned ACT methods. Different methods have been proposed to overcome this problem. One of these methods is the use of heptagonal configuration of transducers [132] which creates a field such that small particles can still be moved to certain locations inside the heptagonal region. The feasibility of the approach was shown on localization of particles as small as $1 \mu \mathrm{m}$. Alternatively, the use of secondary forces were also utilized. Once the bio-particles start the concentrate at certain locations and form clumps, the secondary forces which causes attraction forces at the vicinity of the particles begin to generate. These secondary forces improve the trapping performance. In a recent study, the secondary forces have been used to trap submicron bio-particles [133]. 10-12 $\mu \mathrm{m}$ particles were loaded into the channel prior to the loading of the sub-micron particles. These 
particles created more dominant secondary effect, and an efficient trapping of $490 \mathrm{~nm}$ particles were succeeded (a more detailed review of bio-particle trapping can be found elsewhere [134]). The separation of bio-particles by exploiting the difference in size and acoustic properties has been performed successfully including separation of lipid particles from erythrocytes [69], human cells from bacteria [108], human sperm from epithelial cell DNA [85], separation of plasma from the rest of the blood cells [91,9], removal of platelets from a progenitor cell product [81], separation of cells at different phases of mitosis [135], separation of viable and nonviable mammalian cells from each other [94] and detection of prostate specific antigen (PSA) in whole blood [136].

In comparison to engineered micro-particles, the distributions of size, shape and acoustic properties have larger variations in biological samples which makes the separation process of bio-particles more challenging. One of the major concerns for the bio-particles is that they may be negatively affected from the separation, concentration or washing processes. It is known that cells may be damaged or activated (like platelets) because of shear which occur during processing. Cell viability has also been assessed after acoustic separation or manipulation processes. In order to determine the viability of bio-particles, trypan blue was used, and it was observed that the cells were not negatively affected from acoustic manipulation process $[81,86,92,110]$ as they were viable after being exposed to ultrasonic waves. Referring to the strong suits of the ACT methods, an ACT diagnosis device has already commercialized (Attune Acoustic Focusing Cytometer by Life Sciences, www.lifetechnologies.com).

MG methods for bio-particle manipulation can be grouped into three different types of applications for the clinical and diagnostic applications. The first group focuses on use magnetic field on bio-particles, namely RBCs, which are affected from magnetic field without the use of paramagnetic particles or suspensions. This is due to an exceptional property of deoxygenated RBCs. The hemoglobin protein in an RBC carries iron molecules; hence, will respond to magnetic field if it is deoxygenated. Due to this special property the RBCs can be separated out from a blood sample under the influence of a strong magnetic field in a microchannel. In this respect, the separation of the components of blood through manipulation of RBCs with magnetic field has been implemented $[112,113,138]$. A ferro-mangetic wire in the flow path which was magnetized through an external permanent magnet was able to deflect the RBCs where as the WBCs were not affected from the magnetic field. The capture rate of $90 \%$ (for the RBCs) and throughput in the order of $10^{6}$ cells/minute have been achieved [113]. The change in magnetic susceptibility of RBCs which are affected from the malaria infection, has also been utilized for the separation of infected RBCs [139]. The second group focuses on the diamagnetic manipulation method to focus, trap and separate engineered particles as well as bio-particles. The diamagnetic method requires the engineered or bio-particles to be suspended in a ferro or paramagnetic suspension medium. The yeast cells [140] as well as HaCaT (human skin keratinocyte) cells [76] were successfully focussed to the center of the channel. Trapping and concentration of the yeast cells [141], mouse fibroblast and algea [111] have been achieved. The diamagnetic separation principle can also be applied for separation by size [102]. The yeast cells have been successfully separated from each other in EMG408 ferrofluid [142] with a permanent magnet. Alternating magnetic field can also be applied to separate RBCs and RBCs with sickle cell anemia [65]. The third group focuses on the immunomagnetic bio-particle manipulation for selection of rare cells such as separation of tumor cells from other cell types such as blood cells. Using immunomagnetic labeling of the cancer cells, colon, skin and breast cancer cells have been successfully separated from whole blood [143]. Moreover, the separation of MCF-7, SK-BR-3 breast cancer cell lines from whole blood $[48,99,64]$, capturing of rare colon and breast cancer cells with a very low concentration $\left(10^{9}: 1\right)$ [64], detection and concentration of influenza virus [98], the separation of tagged leukocytes in blood from RBCs with the use of magnetic stripes[144], collection of CD34+ stem cells from leukapheresis product $[70,71]$ using millimeter size tube and a quadrupole magnet arrangement have been successfully performed. Switching of the magnetic field may provide an additional selectivity for separation of bio-particles, and was used for the separation of blood cells and bacteria [65].

Viability is especially important for diamagnetic based manipulation methods since suspending the bio-particles in ferromagnetic or paramagnetic fluids may affect the viability or longevity of bioparticles. The effect of paramagnetic and ferrofluids on cell viability has been investigated, and it was reported that HaCaT cells were half dead after being exposed to paramagnetic fluid for $4 \mathrm{~h} \mathrm{[76].} \mathrm{In}$ another study yeast cells, after being focused in a paramagnetic fluid, were plated in agar plates and the CFU/ml (colony forming unit) was counted [140]. When compared against a control unit, it was observed that only $10 \%$ less colonies were formed compared to control samples. Regarding these studies, it may be concluded that the cells in paramagnetic fluid are affected negatively however the severity of the effect depends on the type of cells, concentration of the paramagnetic fluid, as well as the duration of time which cells were suspended in the medium. Therefore, in terms of viability the diamagnetic manipulation methods may be implemented on diagnosis applications without important negative drawbacks. However for clinical purposes, the suspension of cells in such medium and then infusing it back to the patient may be problematic due to viability issues as well as possibility of allergic reactions to these ferro and paramagnetic fluids.

In OP methods, optical forces generated with a single beam of light that is capable of manipulating a single object is the basic idea behind the optical tweezers. Optical tweezers have been implemented for the separation of a single yeast cell from the population [145], transporting bacteria from the inlet reservoir to other reservoirs containing fluorescent dyes [146], to isolate a single cell and perform single-cell proteomic analysis [147], for the movement of a single cell between two distinct media flow (at the Y-junction of a microfluidic channel) to monitor the cell response to environmental changes [148]. Although the separation and transportation were performed passively and no physical information about the trapped object was used, active devices can be implemented in which certain characteristics of objects are fed back to a control system and decisions are made accordingly for the sorting of biological cells according to their physical and chemical characteristics. Optical forces can also be utilized for sorting in a microfluidic environment by optically deflecting fluorescently labeled cells with a throughput of $\sim 20-100$ cells/s [72]. Optical tweezer based microfluidic sorters have been able to isolate infected mouse macrophages from a population to show its effective use in diagnostics [67], and cells with different sizes using adaptive image processing methods [74,73]. OP methods have been worked extensively, and following the research phase, commercialization of microfluidic based optical trapping technology is underway [149].

Applications that require trapping of multiple particles are achievable by creating multiple optical beams (i.e. focusing on multiple spots). Multiple beam paths can be created by splitting a single beam of light into more beams and forming a spatial pattern. Spatial light modulators are instruments specifically designed to achieve dynamic trapping patterns [150]. Both holographic optical tweezers and generalized phase contrast based optical traps use spatial light modulator technology to create reconfigurable trap sites in 3D space. The applicability of 3D tweezers has been demonstrated to manipulate yeast cells [151] and E. coli bacteria [152] 


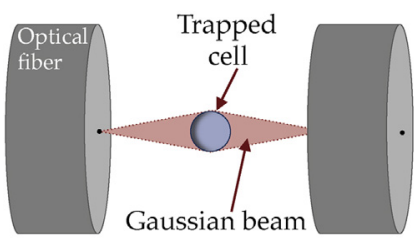

(a)

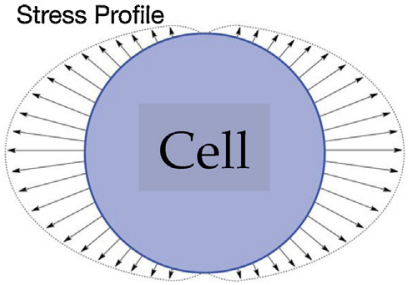

(b)
Fig. 9. (a) Dual-beam optical trap using Gaussian beams launched from optical fiber. (b) Optical stress profile on a biological cell results in elongation.

in a petri-dish environment and in a microfluidic channel [153]. Hexagonal holographic optical trap has been also utilized for the measurement of the tension built up throughout the actin filament network in a controlled chemical microfluidic environment [154].

Mechanical properties of bio-particles play an essential role in for cell biology and diagnostics. Stretching of bio-particles with optical tweezers is a common practice to explore the mechanical properties of bio-particles. Typically, optical tweezers are used in cell-culture plate environment to study erythrocyte biomechanics [54]. A dielectric particle binds to the cell of interest and can be held with a micropipette, while another particle is manipulated using a single focused laser beam. This approach requires mechanical contact to cell, and is low throughput due to simultaneous manipulation of light and micropipette. To overcome such problems, a microfluidic based dual-beam optical trap was proposed to study cells involved in malignant transformation [155]. Healthy, cancerous and metastatic breast epithelial cells were trapped and elongated to compare their deformability. Unlike a single focused beam that attracts the cell, two diverging beams with same spatial characteristics and power were used. A single diverging beam pushes the cell due to higher scattering force while two combined beams can trap the cell at the center of the channel. When trapped, momentum change on the front and back surfaces creates force vectors that do not compress but elongate the cell (see Fig. 9). The advantage of this method is its superior throughput compared to standard optical tweezers although it is still necessary to stop the flow in the channel during the stretching experiment. To overcome this issue, a dynamic approach involving a high power laser diode bar was introduced to study erythrocyte elasticity [156]. This approach took advantage of the fact that a single optical beam also creates instant stretching forces while pushing the cell. Dual-beam optical trap was later applied to characterize mechanical properties of giant unilamellar vesicles, which are model systems for plasma membrane [157], and to detect molecular signatures of polymer network in a biological cell by Raman spectroscopy [158]. More recently, EK techniques have also been implemented to stretch the bio-particles between electrodes. DEP has been utilized to stretch two cell types, one of cancerous origin (MCF-7) and the other from related non-cancerous tissue (MCF-10A) [159]. The results revealed that by analyzing the mechanical behavior of the cells, cell types can be identified.

\section{Hybrid methods}

As discussed so far, each method has its own advantages, disadvantages and limitations in terms of sample preparation, selectivity, throughput, fabrication, etc. Depending on the application one particular method may be superior compared to the others. However, hybrid methods which combine some of these technique may extend the capabilities of each method and offer some new possibilities.

The hybrid implementation of optics with EK (which is also called opto-electric $(\mathrm{OE})$ ) is a relatively mature field (an excellent overview of OE manipulation can be found elsewhere [160,161]). In OE applications, the optics is used to modify and perturb the local electrical properties of a patterned surface. The patterning is performed either by a programmable illumination system or by a mask. If a photoconductive layer such as amorphous silicon (for low conductivity buffer solution, $<100 \mathrm{mS} / \mathrm{m}$ ) or a phototransistor array (for high conductivity buffer solutions, $>1 \mathrm{~S} / \mathrm{m}$ ) is coated on the surface, it is possible to get a desired electrode pattern (i.e. virtual electrodes) on the surface with the aid of optics [160]. With the desired electrode pattern DEP can be utilized for the manipulation of bio-particles. OE based microfluidic systems has proven to manipulate many different types of bio-particles such as RBCs, WBCs, HeLa cells, Jurkat cells, motile and non-motile sperms to name few [161].

ACT methods have a promising throughput with relatively lower selectivity. The selectivity of ACT methods can be improved by combining it with DEP or OP methods. Ravula et al. [162] utilized the ACT method to manipulate large number of particles into a relatively larger streams as pre-concentrator and utilized the DEP for single stream focusing with a throughput of $10^{4}-10^{5}$ particles $/ \mathrm{min}$. The combination of two methods resulted in better focusing with reduced variability in the particles location which would improve the fidelity of the any optical signal based measurement. More recently, an integrated architecture is simulated for the washing, separation and concentration of the particles in which ACT methods are utilized for the washing and concentration, and DEP is utilized for the separation [163]. In another study, ACT trapping has been combined with OP trapping to manipulate larger particles (with an effective diameter $>70 \mu \mathrm{m}$ ) [164]. It is not possible to levitate the large particles with a reasonable laser power within a microchannel; therefore, ACT trapping was used to levitate and trap the particles within a certain focus plane, and a optical tweezers has been utilized to manipulate the particle within this focus plane. The manipulation of living dinoflagellates and potato starch grain has been successfully achieved [164]. EK methods have also combined with MG methods to focus particles [165] and screening of the mechanical properties of lipid bilayer vesicles [166]. In these studies [165,166], different methods are employed to create different forces to manipulate particles in different directions.

\section{Concluding remarks and outlook}

Microfluidics and lab-on-a-chip technology offers unique advantages for the next generation devices for diagnostic and therapeutic applications. Manipulation of the bio-particles is the key ingredient for these applications. Different techniques are available for the manipulation of bio-particles in microfluidic techniques. Some of the techniques together with their strong suits and weaknesses have been discussed. The comparison of different techniques and the recent applications regarding the microfluidic bio-particle manipulation are presented. Some of the major remarks, challenges and future research directions for microfluidic bio-particle manipulation can be summarized as:

- HD methods offer a label-free manipulation with high throughput potential using inertial microfluidics. However, its selectivity is limited to size and shape. Sample preparation may be needed such as the dilution of the particle suspension. The hardware required is flow control devices such as pumps and valves. Fabrication process can be performed using standard microfabrication techniques.

- EK applications offers a label-free manipulation with relatively low-throughput for clinical applications. However, its selectivity is superior; same size, same type cells may be differentiated according to their cell cycle state. The prediction about the 
response of the cells is not straightforward, and sometimes additional effort is required. Typically dilution of the biological buffer solution is necessary. Fabrication requires additional steps if fabrication of the embedded electrodes is necessary. On top of the flow control hardware, AC or DC power supply is required.

- ACT applications offer label-free manipulation with relatively high throughput. Its selectivity depends on the size and the acoustic properties of the particles. However, having the channel geometry is critical for successful operation. On top of the flow control hardware, a piezoelectric material and an AC power supply to actuate the piezoelectric material are required.

- MG applications offer bio-particle manipulation with relatively high throughput. In many cases, the labeling of either the particles or changing the buffer solution is required. With a proper labeling, this method is highly selective. On top of the flow control hardware, a permanent magnet or an electro-magnet is necessary. Due to the labeling requirement, it is not preferable for therapeutic applications.

- OP applications (or optical tweezers) offers high precision manipulation with low throughput applications. OP methods may or may not require labeling. They have an excellent selectivity with proper labeling. Conventional systems do not require any additional fabrication steps. Typically, on top of flow control hardware, a laser and a driver equipment, optomechanical instruments, a microscopy system, photodetectors, an amplifier circuitry, and a motorized stage is required. Microfluidic OP applications is best suited to practical applications involving displacing bio-particles in a flow environment, or precise delivery of biochemical stimuli to bio-particles.

- Regarding OP applications, a research effort needs to be dedicated on building compact optical force instrumentation using optical fibers to avoid the necessity to use a light microscope. This way, optical forces can be harnessed to create smart micro total analysis systems using miniaturized setups which require minimal intervention. The advances in piezo-positioning stages and its control electronics, laser holography and spatial light modulator systems, and fast imaging systems will alleviate the challenges, and possibly open the road for more than proof-of-concept applications.

- High-precision mechanical machining is an alternative method for lithography-based fabrication of the molds. The unique advantage of being able to fabricate microchannels with varying heights and high aspect-ratio, the performance of the microfluidic systems may be improved. Moreover, the hybrid use of CNC machining with lithography-based microfabrication techniques may open up some new opportunities. Commercialization stage of the microfluidic technologies requires the fabrication of microfluidic devices on a mass scale and low cost. Injection molding, which is a well-known manufacturing process for many plastic based devices we used in our daily life, together with the high-precision CNC machining can be utilized for mass-scale production. Once a mold has been manufactured with high precision, several thousand parts can be replicated with little or no extra effort.

- Hybrid methods which combines several methods to compensate the drawback of each method involved in the process is a promising research field. Combination of these methods may extend the capabilities of each method and offer some new possibilities. More research efforts may be expected to be devoted in hybrid methods in near future.

- There exist two clinical applications of aforementioned methods: diagnostics and therapeutic. In diagnostic applications, the amount of cells that need to be processed is significantly lower compared to that of therapeutic application; hence, low throughputs are tolerable. Final biological state of the bio-particles is not a concern as long as the necessary information is extracted from the sample. In therapeutic applications however, the initial biological state of the bio-particles needs to be preserved and the amount of bio-particles to be processed is several order of magnitude higher. Hence, high throughput is extremely important for therapeutic applications. Pre-processing such as dilution, labeling, suspension medium change is not preferable in therapeutic applications. Considering all these constraints, there are limited number of methods promising for therapeutic applications. Regarding their throughputs and the label-free nature, the ACT and HD (more specifically inertial microfluidics) seem to be strong candidates for commercial bio-particle manipulation devices for therapeutic applications in the near future.

- Simulation is important for an efficient design of microfluidics devices for bio-particle separation. Typical microfluidic device the channel length is long compared to the width and height of the channel and this requires large number of mesh within the solution domain. The problem becomes more complicated with the inclusion of multiphysics nature and the presence of the moving particle(s). In some cases, 2D modeling with point particle approach, which neglects the disturbance of the particle on the field, together with some statistical approach may be an assessment tool $[163,167]$. However, point particle approach especially for hydrodynamic application is not preferable since the trajectory of the particle is determined by the shape and the interaction of the particle(s) with the flow field and the walls. Therefore, the development of computational models which can handle particle(s) presence within the solution domain is important for the development of microfluidic technologies.

- In order for a microfluidic bio-particle manipulation prototype (method) to be a successful commercial biomedical or biotech device, certain requirements need to be satisfied. The design needs to be economically feasible (especially true for the disposable components of the design), should allow sterilization of the disposable components and prevent the any contact of biological fluids and cells with the hardware components. Moreover, the materials used needs to be biocompatible, and the interface between the hardware and the disposable set should be simple and intuitive so that the disposable set can be loaded on the hardware reliably by the user.

\section{Acknowledgments}

Dr. Çetin would like to thank Ms. Soheila Zeinali for her assistance in the preparation of 3D drawings, and Dr. Sinan Filiz for the fabrication and preparation of the SEM images of the mold and channels fabricated by micro-machining. Dr. Solmaz would like to acknowledge TÜBITAK 2232 program (113C001) and Katip Çelebi University Scientific Research Project (2014-1-MUH-23) for the financial support.

\section{References}

[1] N.-T. Nguyen, S.T. Wereley, Fundamentals and Applications of Microfluidics, Artech House, Norwood, MA, 2006, pp. 49-50, 67-129.

[2] D. Li, Electrokinetics in Microfluidics, Elsevier Academic Press, London, UK, 2004, pp. 1-5.

[3] D. Erickson, D. Li, Integrated microfluidic devices, Anal. Chim. Acta 507 (2004) $11-26$.

[4] R. Pethig, Dielectrophoresis: an assessment of its potential to aid the research and practice of drug discovery and delivery, Adv. Drug Deliv. Rev. 65 (2013) 1589-1599.

[5] A. Castellanos, A. Ramos, A. Gonzalez, N.G. Green, H. Morgan, Electrohydrodynamics and dielectrophoresis in microsystems: scaling laws, J. Phys. D: Appl. Phys. 36 (2003) 2584-2597.

[6] Z. Wu, K. Hjort, Microfluidic hydrodynamic cell separation: a review, Micro Nanosyst. 1 (3) (2009) 181-192.

[7] R. Pethig, Review article "Dielectrophoresis: status of the theory, technology and applications", Biomicrofluidics 4 (2010) 022811.

[8] B. Cetin, D. Li, Dielectrophoresis in microfluidics technology, Electrophoresis 32 (2011) 2410-2427. 
[9] M. Evander, J. Nilsson, Acoustofluidics 20: applications in acoustic trapping, Lab Chip 12 (22) (2012) 4667-4676.

[10] T. Leong, L. Johansson, P. Juliano, S.L. McArthur, R. Manasseh, Ultrasonic separation of particulate fluids in small and large scale systems: a review, Ind. Eng. Chem. Res. 52 (47) (2013) 16555-16576.

[11] N.-T. Nguyen, Micro-magnetofluidics: interactions between magnetism and fluid flow on the microscale, Microfluid. Nanofluid. 12 (1-4)(2012) 1-16.

[12] L. Borlido, A.M. Azevedo, A.C.A. Roque, M.R. Aires-Barros, Magnetic separations in biotechnology, Biotechnol. Adv. 31 (8) (2013) 1374-1385.

[13] K.C. Neuman, S.M. Block, Optical trapping, Rev. Sci. Instrum. 75 (9) (2004) 2787-2809.

[14] A. Jonas, P.Zemanek, Light at work: the use of optical forces for particle manipulation, sorting, and analysis, Electrophoresis 29 (24) (2008) 4813-4851.

[15] D. Di Carlo, D. Irimia, R.G. Tompkins, M. Toner, Continuous inertial focusing ordering, and separation of particles in microchannels, Proc. Natl. Acad. Sci. U. S. A. 104 (48) (2007) 18892-18897.

[16] L.R. Huang, E.C. Cox, R.H. Austin, J.C. Sturm, Continuous particle separation through deterministic lateral displacement, Science 304 (5673) (2004) 987-990.

[17] J.A. Davis, D.W. Inglis, K.J. Morton, D.A. Lawrence, L.R. Huang, S.Y. Chou, J.C. Sturm, R.H. Austin, Deterministic hydrodynamics: taking blood apart, Proc. Natl. Acad. Sci. U. S. A. 103 (40) (2006) 14779-14784.

[18] S. Sugaya, M. Yamada, M. Seki, Observation of nonspherical particle behaviors for continuous shape-based separation using hydrodynamic filtration, Biomicrofluidics 5 (2)(2011) 24103.

[19] S.H. Holm, J.P. Beech, M.P. Barrett, J.O. Tegenfeldt, Separation of parasites from human blood using deterministic lateral displacement, Lab Chip 11 (2011) 1326-1332.

[20] K.K. Zeming, S. Ranjan, Y. Zhang, Rotational separation of non-spherical bioparticles using I-shaped pillar arrays in a microfluidic device, Nat. Commun. 4 (2013) 1625

[21] S. Choi, J.-K. Park, Continuous hydrophoretic separation and sizing of microparticles using slanted obstacles in a microchannel, Lab Chip 7 (2007) 890-897.

[22] S. Choi, S. Song, C. Choi, J.-K. Park, Continuous blood cell separation by hydrophoretic filtration, Lab Chip 7 (2007) 1532-1538.

[23] S. Choi, S. Song, C. Choi, J.-K. Park, Hydrophoretic sorting of micrometer and submicrometer particles using anisotropic microfluidic obstacles, Anal. Chem. 81 (1) (2009) 50-55.

[24] S. Choi, S. Song, C. Choi, J.-K. Park, Microfluidic self-sorting of mammalian cells to achieve cell cycle synchrony by hydrophoresis, Anal. Chem. 81 (5) (2009) 1964-1968.

[25] S. Choi, J.-K. Park, Tuneable hydrophoretic separation using elastic deformation of poly(dimethylsiloxane), Lab Chip 9 (2009) 1962-1965.

[26] S. Song, S. Choi, Design rules for size-based cell sorting and sheathless cell focusing by hydrophoresis, J. Chromatogr. A 1302 (2013) 191-196.

[27] M. Yamada, M. Nakashima, M. Seki, Pinched flow fractionation: continuous size separation of particles utilizing a laminar flow profile in a pinched microchannel, Anal. Chem. 76 (18) (2004) 5465-5471.

[28] J. Takagi, M. Yamada, M. Yasuda, M. Seki, Continuous particle separation in a microchannel having asymmetrically arranged multiple branches, Lab Chip 5 (2005) 778-784.

[29] M. Yamada, M. Seki, Microfluidic particle sorter employing flow splitting and recombining, Anal. Chem. 78 (4) (2006) 1357-1362.

[30] C. Cupelli, T. Borchardt, T. Steiner, N. Paust, R. Zengerle, M. Santer, Leukocyte enrichment based on a modified pinched flow fractionation approach, Microfluid. Nanofluid. 14 (3-4) (2013) 551-563.

[31] J.F. Ashley, C.N. Bowman, R.H. Davis, Hydrodynamic separation of particles using pinched-flow fractionation, AIChE J. 59 (9) (2013) 3444-3457.

[32] D. Di Carlo, Inertial microfluidics, Lab Chip 9 (2009) 3038-3046.

[33] Z. Wu, B. Willing, J. Bjerketorp, J.K. Jansson, K. Hjort, Soft inertial microfluidics for high throughput separation of bacteria from human blood cells, Lab Chip 9 (2009) 1193-1199.

[34] S.C. Hur, A.J. Mach, D. Di Carlo, High-throughput size-based rare cell enrichment using microscale vortices, Biomicrofluidics 5 (2) (2011) 022206.

[35] J. Autebert, B. Coudert, F.-C. Bidard, J.-Y. Pierga, S. Descroix, L. Malaquin, J.-L. Viovy, Microfluidic: an innovative tool for efficient cell sorting, Methods 57 (3) (2012) 297-307.

[36] M. Masaeli, E. Sollier, H. Amini, W. Mao, K. Camacho, N. Doshi, S. Mitragotri, A Alexeev, D. Di Carlo, Continuous inertial focusing and separation of particles by shape, Phys. Rev. X 2 (2012) 031017.

[37] H. Amini, E. Sollier, M. Masaeli, Y. Xie, B. Ganapathysubramanian, H.A. Stone, D. Di Carlo, Engineering fluid flow using sequenced microstructures, Nat. Commun. 4 (2013) 1826.

[38] A.J. Chung, D.R. Gossett, D. Di Carlo, Microfluidics: three dimensional, sheathless, and high-throughput microparticle inertial focusing through geometry-induced secondary flows, Small 9 (5) (2013) 804.

[39] A.J. Chung, D. Pulido, J.C. Oka, H. Amini, M. Masaeli, D. Di Carlo, Microstructureinduced helical vortices allow single-stream and long-term inertial focusing, Lab Chip 13 (2013) 2942-2949.

[40] M. Masaeli, E. Sollier, H. Amini, W. Mao, K. Camacho, N. Doshi, S. Mitragotri, A. Alexeev, D. Di Carlo, Continuous inertial focusing and separation of particles by shape, Phys. Rev. X 2 (2012) 031017.

[41] J. Voldman, Electrical forces for microscale cell manipulation, Annu. Rev. Biomed. Eng. 8 (2006) 425-454.
[42] B. Cetin, D. Li, Effect of Joule heating on electrokinetic transport, Electrophoresis 29 (2008) 994-1005.

[43] R.C. Gallo-Villanueva, C.E. Rodriguez-Lopez, R.I. Diaz-de-la Garza, C. Reyes-Betanzo, B.H. Lapizco-Encinas, DNA manipulation by means of insulator-based dielectrophoresis employing direct current electric fields, Electrophoresis 30 (24) (2009) 4195-4205.

[44] L.V. King, On the acoustic radiation pressure on spheres, Proc. R. Soc. Lond. A 147 (861) (1934) 212-240.

[45] K. Yosioka, Acoustic radiation pressure on a compressible sphere, Acustica 5 (1955) 167-173.

[46] L.P. Gor'Kov, On the forces acting on a small particle in an acoustical field in an ideal fluid, Sov. Phys. Dokl. 6 (1962) 773.

[47] H. Bruus, Acoustofluidics 2: perturbation theory and ultrasound resonance modes, Lab Chip 12 (1) (2012) 20-28.

[48] T.P. Forbes, S.P. Forry, Microfluidic magnetophoretic separations of immunomagnetically labeled rare mammalian cells, Lab Chip 12 (8) (2012) 1471-1479.

[49] A. Ashkin, Acceleration and trapping of particles by radiation pressure, Phys Rev. Lett. 24 (4) (1970) 156

[50] S. Chu, J.E. Bjorkholm, A. Ashkin, A. Cable, Experimental observation of optically trapped atoms, Phys. Rev. Lett. 57 (3) (1986) 314.

[51] A. Ashkin, J.M. Dziedzic, Optical trapping and manipulation of viruses and bacteria, Science 235 (4795) (1987) 1517-1520.

[52] J.R. Moffitt, Y.R. Chemla, S.B. Smith, C. Bustamante, Recent advances in optical tweezers, Annu. Rev. Biochem. 77 (2008) 205-228.

[53] D.G. Grier, Optical tweezers in colloid and interface science, Curr. Opin. Colloid Interface Sci. 2 (3) (1997) 264-270.

[54] M. Dao, C.T. Lim, S. Suresh, Mechanics of the human red blood cell deformed by optical tweezers, J. Mech. Phys. Solids 51 (11) (2003) 2259-2280.

[55] Z. Bryant, M.D. Stone, J. Gore, S.B. Smith, N.R. Cozzarelli, C. Bustamante, Structural transitions and elasticity from torque measurements on DNA, Nature 424 (6946) (2003) 338-341.

[56] A. Ashkin, Forces of a single-beam gradient laser trap on a dielectric sphere in the ray optics regime, Biophys. J. 61 (2) (1992) 569-582.

[57] G. Roosen, C. Imbert, Optical levitation by means of two horizontal laser beams: a theoretical and experimental study, Phys. Lett. A 59 (1) (1976) 6-8.

[58] A. Ashkin, J.M. Dziedzic, J.E. Bjorkholm, S. Chu, Observation of a single-beam gradient force optical trap for dielectric particles, Opt. Lett. 11 (5) (1986) 288-290.

[59] A.C. Sabuncu, J. Zhuang, J.F. Kolb, A. Beskok, Microfluidic impedance spectroscopy as a tool for quantitative biology and biotechnology, Biomicrofluidics 6 (3) (2012) 034103

[60] M.W. Stacey, A.C. Sabuncu, A. Beskok, Dielectric characterization of costa cartilage chondrocytes, Biochim. Biophys. Acta 1840 (1) (2014) 146-152.

[61] F. Petersson, L. Åberg, A.-M. Swärd-Nilsson, T. Laurell, Free flow acoustophoresis: microfluidic-based mode of particle and cell separation, Anal. Chem. 79 (14) (2007) 5117-5123.

[62] M.A. Burguillos, C. Magnusson, M. Nordin, A. Lenshof, P. Augustsson, M.J. Hansson, E. Elmer, H. Lilja, P. Brundin, T. Laurell, T. Deierborg, Microchanne acoustophoresis does not impact survival or function of microglia, leukocytes or tumor cells, PLOS ONE 8 (5) (2013) e64233.

[63] K.W. Cushing, M.E. Piyasena, N.J. Carroll, G.C. Maestas, B.A. Lopez, B.S Edwards, S.W. Graves, G.P. Lopez, Elastomeric negative acoustic contrast particles for affinity capture assays, Anal. Chem. 85 (4) (2013) 2208-2215.

[64] K. Hoshino, Y.-Y. Huang, N. Lane, M. Huebschman, J.W. Uhr, E.P. Frenkel, $\mathrm{X}$. Zhang, Microchip-based immunomagnetic detection of circulating tumor cells, Lab Chip 11 (20) (2011) 3449-3457.

[65] A.R. Kose, B. Fischer, L. Mao, H. Koser, Label-free cellular manipulation and sorting via biocompatible ferrofluids, Proc. Natl. Acad. Sci. U.S.A. 106 (51) (2009) 21478-21483.

[66] J. Verbarg, K. Kamgar-Parsi, A.R. Shields, P.B. Howell, F.S. Ligler, Spinning magnetic trap for automated microfluidic assay systems, Lab Chip 12 (10) (2012) 1793-1799.

[67] T.D. Perroud, J.N. Kaiser, J.C. Sy, T.W. Lane, C.S. Branda, A.K. Singh, K.D. Patel Microfluidic-based cell sorting of Francisella tularensis infected macrophages using optical forces, Anal. Chem. 80 (16) (2008) 6365-6372.

[68] M. Li, W.H. Li, J. Zhang, G. Alici, W. Wen, A review of microfabrication techniques and dielectrophoretic micro devices for particle manipulation and separation, J. Phys. D: Appl. Phys. 47 (2014) 063001

[69] J.D. Adams, C.L. Ebbesen, R. Barnkob, A.H.J. Yang, H.T. Soh, H. Bruus, Highthroughput, temperature-controlled microchannel acoustophoresis device made with rapid prototyping, J. Micromech. Microeng. 22 (7) (2012) 075017.

[70] Y. Jing, R.M. Lee, P.S. Williams, J.J. Chalmers, S.S. Farag, B. Bolwell, M Zborowski, Blood progenitor cell separation from clinical leukapheresis product by magnetic nanoparticle binding and magnetophoresis, Biotechnol. Bioeng. 96 (6) (2007) 1139-1154.

[71] R.M. Lee, R.R. Alexander, P.S. Williams, K. McCloskey, J.B. Brian, M. Nakamura, J.J. Chalmers, M. Zborowski, Progenitor cell isolation with a high-capacity quadrupole magnetic flow sorter, J. Magn. Magn. Mater. 225 (1) (2001) 277-284.

[72] M.W. Mark, E. Tu, D.E. Raymond, J.M. Yang, H. Zhang, N. Hagen, B. Dees, E.M. Mercer, A.H. Forster, I. Kariv, J.M. Philippe, F.B. William, Microfluidic sorting of mammalian cells by optical force switching, Nat. Biotechnol. 23 (1) (2005) 83-87. 
[73] X. Wang, S. Chen, M. Kong Z. Wang, K.D. Costa, R.A. Li, D. Sun, Enhanced cell sorting and manipulation with combined optical tweezer and microfluidic chip technologies, Lab Chip 11 (21) (2011) 3656-3662.

[74] B. Landenberger, H. Hşšfemann, S. Wadle, A. Rohrbach, Microfluidic sorting of arbitrary cells with dynamic optical tweezers, Lab Chip 12 (17) (2012) 3177-3183.

[75] S.A. Peyman, E.Y. Kwan, O. Margarson, A. Iles, N. Pamme, Diamagnetic repulsion - a versatile tool for label-free particle handling in microfluidic devices, J. Chromatogr. A 1216 (52) (2009) 9055-9062.

[76] A.I. Rodríguez-Villarreal, M.D. Tarn, L.A. Madden, J.B. Lutz, J. Greenman, J. Samitier, N. Pamme, Flow focussing of particles and cells based on their intrinsic properties using a simple diamagnetic repulsion setup, Lab Chip 11 (7) (2011) 1240-1248.

[77] Y.-S. Lin, C.-H. Yang, C.-Y. Wang, F.-R. Chang, K.-S. Huang, W.-C. Hsieh, An aluminum microfluidic chip fabrication using a convenient micromilling process for fluorescent poly(DL-lactide-co-glycolide) microparticle generation, Sensors 12 (2012) 1455-1467

[78] H. Becker, L.E. Locascio, Polymer microfluidic devices, Talanta 56 (2002) 267-287.

[79] B. Cetin, Y. Kang, Z. Wu, D. Li, Continuous particle separation by size via AC-dielectrophoresis using a lab-on-a-chip device with 3D electrodes, Electrophoresis 30 (2009) 766-772.

[80] B. Cetin, D. Li, Lab-on-a-chip device for continuous particle and cell separation based on electrical properties via ac-dielectrophoresis, Electrophoresis 31 (2010) 3035-3043.

[81] J. Dykes, A. Lenshof, B. Åstrand-Grundström, T. Laurell, S. Scheding, Efficien removal of platelets from peripheral blood progenitor cell products using a novel micro-chip based acoustophoretic platform, PLoS ONE 6 (8) (2011) e23074

[82] M. Hill, Y. Shen, J.J. Hawkes, Modelling of layered resonators for ultrasonic separation, Ultrasonics 40 (1) (2002) 385-392.

[83] F. Petersson, A. Nilsson, C. Holm, H. Jönsson, T. Laurell, Continuous separation of lipid particles from erythrocytes by means of laminar flow and acoustic standing wave forces, Lab Chip 5 (1) (2005) 20-22.

[84] J.J. Hawkes, D. Barrow, W.T. Coakley, Microparticle manipulation in millimetre scale ultrasonic standing wave chambers, Ultrasonics 36 (9) (1998) 925-931.

[85] J.V. Norris, M. Evander, K.M. Horsman-Hall, J. Nilsson, T. Laurell, J.P. Landers, Acoustic differential extraction for forensic analysis of sexual assaul evidence, Anal. Chem. 81 (15) (2009) 6089-6095.

[86] A. Haake, A. Neild, D.-H. Kim, J.-E. Ihm, Y. Sun, J. Dual, B.-K. Ju, Manipulation of cells using an ultrasonic pressure field, Ultrasound Med. Biol. 31 (6) (2005) 857-864.

[87] T. Franke, S. Braunmüller, L. Schmid, A. Wixforth, D.A. Weitz, Surface acoustic wave actuated cell sorting (SAWACS), Lab Chip 10 (6) (2010) 789-794.

[88] J. Shi, D. Ahmed, X. Mao, S.-C. Steven Lin, A. Lawit, T.J. Huang, Acoustic tweezers: patterning cells and microparticles using standing surface acoustic waves (SSAW), Lab Chip 9 (20) (2009) 2890-2895.

[89] L. Meng, F. Cai, Q. Jin, L. Niu, C. Jiang, Z. Wang, J. Wu, H. Zheng, Acoustic alignin and trapping of microbubbles in an enclosed PDMS microfluidic device, Sens. Actuators B 160 (1) (2011) 1599-1605.

[90] J.J. Hawkes, R.W. Barber, D.R. Emerson, W.T. Coakley, Continuous cell washing and mixing driven by an ultrasound standing wave within a microfluidic channel, Lab Chip 4 (5) (2004) 446-452.

[91] I. Leibacher, S. Schatzer, J. Dual, Impedance matched channel walls in acoustofluidic systems, Lab Chip 14 (3) (2014) 463-470.

[92] M. Evander, L. Johansson, T. Lilliehorn, J. Piskur, M. Lindvall, S. Johansson, M. Almqvist, T. Laurell, J. Nilsson, Noninvasive acoustic cell trapping in microfluidic perfusion system for online bioassays, Anal. Chem. 79 (7) (2007) $2984-2991$.

[93] I. González, L.J. Fernández, T.E. Gómez, J. Berganzo, J.L. Soto, A. Carrato, A polymeric chip for micromanipulation and particle sorting by ultrasounds based on a multilayer configuration, Sens. Actuators B 144 (1) (2010) 310-317.

[94] H.J. Allen, H. Yang, T. Soh, Acoustophoretic sorting of viable mammalian cells in a microfluidic device, Anal. Chem. 84 (24) (2012) 10756-10762.

[95] A. Mueller, A. Lever, T.V. Nguyen, J. Comolli, J. Fiering, Continuous acoustic separation in a thermoplastic microchannel, J. Micromech. Microeng. 23 (12) (2013) 125006.

[96] F. Petersson, A. Nilsson, H. Jönsson, T. Laurell, Carrier medium exchang through ultrasonic particle switching in microfluidic channels, Anal. Chem. 77 (5) (2005) 1216-1221.

[97] L. Johansson, F. Nikolajeff, S. Johansson, S. Thorslund, On-chip fluorescenceactivated cell sorting by an integrated miniaturized ultrasonic transducer, Anal. Chem. 81 (13) (2009) 5188-5196.

[98] L.-Y. Hung, T.-B. Huang, Y.-C. Tsai, C.-S. Yeh, H.-Y. Lei, G.-B. Lee, A microfluidic immunomagnetic bead-based system for the rapid detection of influenz infections: from purified virus particles to clinical specimens, Biomed. Microdevices 15 (3) (2013) 539-551.

[99] D. Issadore, H. Shao, J. Chung, A. Newton, M. Pittet, R. Weissleder, H. Lee, Selfassembled magnetic filter for highly efficient immunomagnetic separation, Lab Chip 11 (1) (2011) 147-151.

[100] H. Lee, A.M. Purdon, R.M. Westervelt, Manipulation of biological cells using a microelectromagnet matrix, Appl. Phys. Lett. 85 (6) (2004) 1063-1065.

[101] L. Liang, X. Xuan, Diamagnetic particle focusing using ferromicrofluidics with a single magnet, Microfluid. Nanofluid. 13 (4) (2012) 637-643.
[102] L. Liang, X. Xuan, Continuous sheath-free magnetic separation of particles in a U-shaped microchannel, Biomicrofluidics 6 (4) (2012) 044106.

[103] B. Çetin, M.D. Asik, S. Taze, Design and fabrication of a microfluidic device for synthesis of chitosan nanoparticles, J. Nanotechnol. Eng. Med. 4 (3) (2014) 031004.

[104] B. Çetin, M.B. Özer, S. Zeinali, S. Büyükkoçak, Fabrication of microfluidic devices for dielectrophoretic and acoustophoretic applications using high-precision machining, in: In 16th International Conference on Machine Design and Production (UMTIK-2014), 2014

[105] A.C. Sabuncu, J. Zhuang, J.F. Kolb, A. Beskok, Microfluidic impedance spectroscopy as a tool for quantitative biology and biotechnology, Biomicrofluidics 6 (3) (2012) 034103.

[106] F.F. Becker, X.-B. Wang, Y. Huang, R. Pethig, J. Vykoukal, P.R. Gascoyne, Separation of human breast cancer cells from blood by differential dielectric affinity, Proc. Natl. Acad. Sci. U.S.A. 92 (3) (1995) 860-864

[107] T. Zhu, R. Cheng, L. Mao, Focusing microparticles in a microfluidic channel with ferrofluids, Microfluid. Nanofluid. 11 (6) (2011) 695-701.

[108] M. Kumar, D.L. Feke, J.M. Belovich, Fractionation of cell mixtures using acoustic and laminar flow fields, Biotechnol. Bioeng. 89 (2) (2005) 129-137.

[109] A.T. Islam, A.H. Siddique, T.S. Ramulu, V. Reddy, Y.-J. Eu, S.H. Cho, C. Kim, Ultrasonic alignment of bio-functionalized magnetic beads and live cells in PDMS micro-fluidic channel, Biomed. Microdevices 14 (6) (2012) 1077-1084.

[110] M.S. Limaye, W.T. Coakley, Clarification of small volume microbial suspensions in an ultrasonic standing wave, J. Appl. Microbiol. 84 (6) (1998) $1035-1042$.

[111] A. Winkleman, K.L. Gudiksen, D. Ryan, G.M. Whitesides, D. Greenfield, M. Prentiss, A magnetic trap for living cells suspended in a paramagnetic buffer Appl. Phys. Lett. 85 (12) (2004) 2411-2413.

[112] K.-H. Han, A.B. Frazier, Paramagnetic capture mode magnetophoretic microseparator for high efficiency blood cell separations, Lab Chip 6 (2) (2006) 265-273.

[113] H.-K. Seo, Y.-H. Kim, H.-O. Kim, Y.-J. Kim, Hybrid cell sorters for on-chip cell separation by hydrodynamics and magnetophoresis, J. Micromech. Microeng. 20 (9) (2010) 095019

[114] T. Masuda, M. Niimi, H. Nakanishi, Y. Yamanishi, F. Arai, Cancer cell separato using size-dependent filtration in microfluidic chip, Sens. Actuators B 185 (2013) 245-251.

[115] Z. Geng, Y. Ju, W. Wang, Z. Li, Continuous blood separation utilizing spira filtration microchannel with gradually varied width and micro-pillar array, Sens. Actuators B 180 (2013) 122-129.

[116] K.-A. Hyun, K. Kwon, H. Han, S.-I. Kim, H.-I. Jung, Microfluidic flow fractionation device for label-free isolation of circulating tumor cells (CTCS) from breast cancer patients, Biosens. Bioelectron. 40 (1) (2013) 206-212.

[117] M.G. Lee, J.H. Shin, S. Choi, J.-K. Park, Enhanced blood plasma separation by modulation of inertial lift force, Sens. Actuators B 190 (2014) 311-317.

[118] J. Riordon, M. Nash, M. Calderini, M. Godin, Using active microfluidic flow focusing to sort particles and cells based on high-resolution volume measurements, Microelectron. Eng. 118 (2014) 35-40.

[119] H. Yin, D. Marshall, Microfluidics for single cell analysis, Curr. Opin. Biotechnol. 23 (1) (2012) 110-119.

[120] L. Bell, A. Seshia, D. Lando, E. Laue, M. Palayret, S.F. Lee, D. Klenerman, A microfluidic device for the hydrodynamic immobilisation of living fission yeast cells for super-resolution imaging, Sens. Actuators B 192 (2014) $36-41$

[121] S. van den Driesche, V. Rao, D. Puchberger-Enengl, W. Witarski, M.J. Vellekoop, Continuous cell from cell separation by traveling wave dielectrophoresis, Sens. Actuators B 170 (2012) 207-214.

[122] S.K. Ameri, P.K. Singh, M.R. Dokmeci, A. Khademhosseini, Q. Xu, S.R. Sonkusale, All electronic approach for high-throughput cell trapping and lysis with electrical impedance monitoring, Biosens. Bioelectron. 54 (2014) 462-467.

[123] M. Javanmard, S. Emaminejad, C. Gupta, J. Provine, R.W. Davis, R.T. Howe Depletion of cells and abundant proteins from biological samples by enhanced dielectrophoresis, Sens. Actuators B 193 (2014) 918-924.

[124] Y.-L. Deng, J.-S. Chang, Y.-J. Juang, Separation of microalgae with different lipid contents by dielectrophoresis, Bioresour. Technol. 135 (2013) 137-141.

[125] P. Augustsson, L.B. Åberg, A.-M.K. Swärd-Nilsson, T. Laurell, Buffer medium exchange in continuous cell and particle streams using ultrasonic standing wave focusing, Mikrochim. Acta 164 (3-4) (2009) 269-277.

[126] D. Bazou, A. Castro, M. Hoyos, Controlled cell aggregation in a pulsed acoustic field, Ultrasonics 52 (7) (2012) 842-850.

[127] R. Walker, I. Gralinski, K.K. Lay, T. Alan, A. Neild, Particle manipulation using an ultrasonic micro-gripper, Appl. Phys. Lett. 101 (16) (2012) 163504.

[128] A.L. Bernassau, P.G.A. MacPherson, J. Beeley, B.W. Drinkwater, D.R.S. Cumming, Patterning of microspheres and microbubbles in an acoustic tweezers, Biomed. Microdevices (2013) 289-297.

[129] M. Wiklund, S. Nilsson, H.M. Hertz, Ultrasonic trapping in capillaries for traceamount biomedical analysis, J. Appl. Phys. 90 (1) (2001) 421-426.

[130] B. Hammarström, M. Evander, H. Barbeau, M. Bruzelius, J. Larsson, T. Laurell, J. Nilsson, Non-contact acoustic cell trapping in disposable glass capillaries, Lab Chip 10 (17) (2010) 2251-2257.

[131] K.H. Lam, H.-S. Hsu, Y. Li, C. Lee, A. Lin, Q. Zhou, E.S. Kim, K.K. Shung, Ultrahigh frequency lensless ultrasonic transducers for acoustic tweezers application, Biotechnol. Bioeng. 110 (3) (2013) 881-886

[132] M. Nordin, T. Laurell, Two-hundredfold volume concentration of dilute cel and particle suspensions using chip integrated multistage acoustophoresis, Lab Chip 12 (22) (2012) 4610-4616. 
[133] A.L. Bernassau, P. Glynne-Jones, F. Gesellchen, M. Riehle, M. Hill, D.R.S. Cumming, Controlling acoustic streaming in an ultrasonic heptagonal tweezers with application to cell manipulation, Ultrasonics (2013) 268-274.

[134] B. Hammarström, T. Laurell, J. Nilsson, Seed particle-enabled acoustic trapping of bacteria and nanoparticles in continuous flow systems, Lab Chip 12 (21) (2012) 4296-4304.

[135] A. Lenshof, A. Ahmad-Tajudin, K. JŠrås, A.-M. SwŠrd-Nilsson, L. Åberg, G Marko-Varga, J. Malm, H. Lilja, T. Laurell, Acoustic whole blood plasmapheresis chip for prostate specific antigen microarray diagnostics, Anal. Chem. 81 (15) (2009) 6030-6037.

[136] P. Thvoz, J.D. Adams, H. Shea, H. Bruus, H.T. Soh, Acoustophoretic synchronization of mammalian cells in microchannels, Anal. Chem. 82 (7) (2010) 3094-3098.

[137] H. Jönsson, C. Holm, A. Nilsson, F. Petersson, P. Johnsson, T. Laurell, Particle separation using ultrasound can radically reduce embolic load to brain after cardiac surgery, Ann. Thorac. Surg. 78 (5) (2004) 1572-1577.

[138] A. Ki-Ho Han, B. Frazier, Continuous magnetophoretic separation of blood cells in microdevice format, J. Appl. Phys. 96 (10) (2004) 5797-5802

[139] L.R. Moore, H. Fujioka, P.S. Williams, J.J. Chalmers, B. Grimberg, P.A. Zimmerman, M. Zborowski, Hemoglobin degradation in malaria-infected erythrocytes determined from live cell magnetophoresis, FASEB J. 20 (6) (2006) 747-749.

[140] J. Zeng, C. Chen, P. Vedantam, V. Brown, T.-R.J. Tzeng, X. Xuan, Threedimensional magnetic focusing of particles and cells in ferrofluid flow through a straight microchannel, J. Micromech. Microeng. 22 (10)(2012) 105018.

[141] J. Zeng, C. Chen, P. Vedantam, T.-R. Tzeng, X. Xuan, Magnetic concentration of particles and cells in ferrofluid flow through a straight microchannel using attracting magnets, Microfluid. Nanofluid. 15 (1) (2013) 49-55.

[142] J. Zeng, Y. Deng, P. Vedantam, T.-R. Tzeng, X. Xuan, Magnetic separation of particles and cells in ferrofluid flow through a straight microchannel using two offset magnets, J. Magn. Magn. Mater. 346 (2013) 118-123.

[143] C.-H. Wu, Y.-Y. Huang, P. Chen, K. Hoshino, H. Liu, E.P. Frenkel, J.X.J. Zhang K.V. Sokolov, Versatile immunomagnetic nanocarrier platform for capturing cancer cells, ACS Nano 7 (10) (2013) 8816-8823.

[144] D.W. Inglis, R. Riehn, R.H. Austin, J.C. Sturm, Continuous microfluidic immunomagnetic cell separation, Appl. Phys. Lett. 85 (21) (2004) 5093-5095.

[145] F. Arai, A. Ichikawa, M. Ogawa, T. Fukuda, K. Horio, K. Itoigawa, High-speed separation system of randomly suspended single living cells by laser trap and dielectrophoresis, Electrophoresis 22 (2) (2001) 283-288.

[146] J. Enger, M. Goksör, K. Ramser, P. Hagberg, D. Hanstorp, Optical tweezers applied to a microfluidic system, Lab Chip 4 (3) (2004) 196-200.

[147] W. Hellmich, C. Pelargus, K. Leffhalm, A. Ros, D. Anselmetti, Single cell manipulation, analytics, and label-free protein detection in microfluidic devices for systems nanobiology, Electrophoresis 26 (19) (2005) 3689-3696.

[148] E. Eriksson, J. Enger, B. Nordlander, N. Erjavec, K. Ramser, M. Goksšr, S. Hohmann, T. Nystršm, D. Hanstorp, A microfluidic system in combination with optical tweezers for analyzing rapid and reversible cytological alterations in single cells upon environmental changes, Lab Chip 7 (1) (2007) $71-76$.

[149] T. Roth, J. Glückstad, Bio optofluidics cell sorter: cell-bocs concept and applications, SPIE 8274 (2012).

[150] E. Schonbrun, R. Piestun, P. Jordan, J. Cooper, K. Wulff, J. Courtial, M. Padgett, $3 \mathrm{~d}$ interferometric optical tweezers using a single spatial light modulator, Opt. Express 13 (10) (2005) 3777-3786.
[151] P.J. Rodrigo, V.R. Daria, J. Glückstad, Real-time three-dimensional optical micromanipulation of multiple particles and living cells, Opt. Lett. 29 (19) (2004) 2270-2272.

[152] P. Jordan, J. Leach, M. Padgett, P. Blackburn, N. Isaacs, M. Goksšr, D. Hanstorp, A. Wright, J. Girkin, J. Cooper, Creating permanent 3d arrangements of isolated cells using holographic optical tweezers, Lab Chip 5 (11) (2005) 1224-1228.

[153] I.R. Perch-Nielsen, P.J. Rodrigo, C.A. Alonzo, J. Glückstad, Autonomous and 3d real-time multi-beam manipulation in a microfluidic environment, Opt Express 14 (25) (2006) 12199-12205.

[154] K. Uhrig, R. Kurre, C. Schmitz, J.E. Curtis, T. Haraszti, A.E.M. Clemen, J.P. Spatz, Optical force sensor array in a microfluidic device based on holographic optical tweezers, Lab Chip 9 (5) (2009) 661-668.

[155] J. Guck, S. Schinkinger, B. Lincoln, F. Wottawah, S. Ebert, M. Romeyke, D. Lenz, H.M. Erickson, R. Ananthakrishnan, D. Mitchell, Optical deformability as an inherent cell marker for testing malignant transformation and metastatic competence, Biophys. J. 88 (5) (2005) 3689-3698.

[156] I. Sraj, C.D. Eggleton, R. Jimenez, E. Hoover, J. Squier, J. Chichester, D.W.M. Marr Cell deformation cytometry using diode-bar optical stretchers, J. Biomed. Opt. 15 (4) (2010) 047010.

[157] M.E. Solmaz, S. Sankhagowit, R. Biswas, C.A. Mejia, M.L. Povinelli, N. Malmstadt, Optical stretching as a tool to investigate the mechanical properties of lipid bilayers, RSC Adv. 3 (37) (2013) 16632-16638.

[158] P.R.T. Jess, V. Garceś-Chvez, D. Smith, M. Mazilu, L. Paterson, A. Riches, C.S Herrington, W. Sibbett, K. Dholakia, Dual beam fibre trap for raman microspectroscopy of single cells, Opt. Express 14 (12)(2006) 5779-5791.

[159] I. Guido, C. Xiong, M.S. Jaeger, C. Duschl, Microfluidic system for cell mechanics analysis through dielectrophoresis, Microelectron. Eng. 97 (2012) 379-382.

[160] A. Kumar, S.J. Williams, H.-S. Chuang, N.G. Green, S.T. Wereley, Hybrid optoelectric manipulation in microfluidics - opportunities and challenges, Lab Chip 11 (2011) 2135-2148.

[161] H. Hwang, J.-K. Park, Optoelectrofluidic platforms for chemistry and biology, Lab Chip 11 (2011) 33-47.

[162] S.K. Ravula, D.W. Branch, C.D. James, R.T. Townsend, M. Hill, G. Kaduchak M. Ward, I. Brener, A microfluidic system combining acoustic and dielectrophoretic particle preconcentration and focusing, Sens. Actuators B 130 (2008) 645-652.

[163] B. Çetin, S. Büyükkoçak, S. Zeinali, M.B. Özer, Simulation of an integrated microfluidic device for bioparticle wash, separation and concentration, in ASME 4th Microscale/Nanoscale Heat \& Mass Transfer International Conference, December 11-14, Hong Kong, China, 2013.

[164] G. Thalhammer, R. Steiger, M. Meinschad, M. Hill, S. Bernet, M. Ritsch-Marte Combined acoustic and optical trapping, Biomed. Opt. Express 2 (October (10)) (2011) 2859-2870.

[165] C.D. James, J. McClain, K.R. Pohl, N. Reuel, K.E. Achyuthan, C.J. Bourdon, K. Rahimian, P.C. Galambos, G. Ludwig, M.S. Derzon, High-efficiency magnetic particle focusing using dielectrophoresis and magnetophoresis in a microfluidic device, J. Micromech. Microeng. 20 (4) (2010) 045015

[166] D. Issadore, T. Franke, K.A. Brown, T.P. Hunt, R.M. Westervelt, Highvoltage dielectrophoretic and magnetophoretic hybrid integrated circuit/microfluidic chip, J. Microelectromech. Syst. 18 (6) (2009) 1220-1225.

[167] S. Büyükkoçak, M.B. Özer, B. Çetin, Numerical modeling of acoustophoretic particle separation for microfuildics, Microfluid. Nanofluid. (2014) http://dx.doi.org/10.1007/s10404-014-1398-7 (in press). 